\title{
Numerical and Experimental Dynamic Analysis of IC Engine Test Beds Equipped with Highly Flexible Couplings
}

\author{
M. Cocconcelli, ${ }^{1}$ M. Troncossi, ${ }^{2}$ E. Mucchi, ${ }^{3}$ A. Agazzi, ${ }^{3}$ A. Rivola, ${ }^{2}$ \\ R. Rubini, ${ }^{1}$ and G. Dalpiaz ${ }^{3}$ \\ ${ }^{1}$ Department of Science and Engineering Methods, University of Modena and Reggio Emilia, \\ Via G. Amendola 2 Pad. Morselli, 42122 Reggio Emilia, Italy \\ ${ }^{2}$ Department of Industrial Engineering, University of Bologna, Via Fontanelle 40, 47121 Forli, Italy \\ ${ }^{3}$ Department of Engineering, University of Ferrara, Via Saragat 1, 42122 Ferrara, Italy \\ Correspondence should be addressed to E. Mucchi; emiliano.mucchi@unife.it
}

Received 14 February 2017; Revised 27 April 2017; Accepted 8 May 2017; Published 9 July 2017

Academic Editor: Pedro Galvín

Copyright (C) 2017 M. Cocconcelli et al. This is an open access article distributed under the Creative Commons Attribution License, which permits unrestricted use, distribution, and reproduction in any medium, provided the original work is properly cited.

\begin{abstract}
Driveline components connected to internal combustion engines can be critically loaded by dynamic forces due to motion irregularity. In particular, flexible couplings used in engine test rig are usually subjected to high levels of torsional oscillations and time-varying torque. This could lead to premature failure of the test rig. In this work an effective methodology for the estimation of the dynamic behavior of highly flexible couplings in real operational conditions is presented in order to prevent unwanted halts. The methodology addresses a combination of numerical models and experimental measurements. In particular, two mathematical models of the engine test rig were developed: a torsional lumped-parameter model for the estimation of the torsional dynamic behavior in operative conditions and a finite element model for the estimation of the natural frequencies of the coupling. The experimental campaign addressed torsional vibration measurements in order to characterize the driveline dynamic behavior as well as validate the models. The measurements were achieved by a coder-based technique using optical sensors and zebra tapes. Eventually, the validated models were used to evaluate the effect of design modifications of the coupling elements in terms of natural frequencies (torsional and bending), torsional vibration amplitude, and power loss in the couplings.
\end{abstract}

\section{Introduction}

Flexible couplings enable the transmission of torque from a driver to a driven part of rotating equipment, by accommodating a certain amount of shaft misalignment. This is obtained by reducing the reaction forces due to axial, lateral, angular displacements that are usually present between the coupled shafts. Flexible couplings with torsional compliance (also known as highly flexible couplings) are used to reduce the transmission of shock loads from one shaft to another and/or to alter the elastodynamic characteristics of the driveline by controlling the natural frequencies of the rotating units. The literature is rich of research works regarding highly flexible couplings, with particular reference to installation in test bench for automotive applications, as in the work of Rabeih and Crolla [1] or Reitz et al. [2]. In particular, Gequn et al. [3] performed the dynamic analysis of the driveline-including a multibody model of the coupling-in order to estimate the influence of bench installation condition on the engine main bearing load. The dynamic behavior of driveline including flexible couplings was also challenged from experimental standpoints, trying to evaluate the degree of misalignment of the coupled shafts; the papers of Dewell and Mitchell [4], Cho and Jeong [5], and Patel and Darpe [6] present good descriptions of such a scenario. The same issue was addressed by Xu and Marangoni [7], using an analytical approach based on the Component Mode Synthesis method. Cruz-Peragón et al. [8] investigated the nonlinear behavior of highly flexible couplings and proposed a methodology based on numerical models and experimental measurements in order to estimate the main coupling parameters.

In this work, the dynamic analysis of the coupling elements in internal combustion (IC) engine test rigs is accounted from both the numerical and experimental standpoints. 


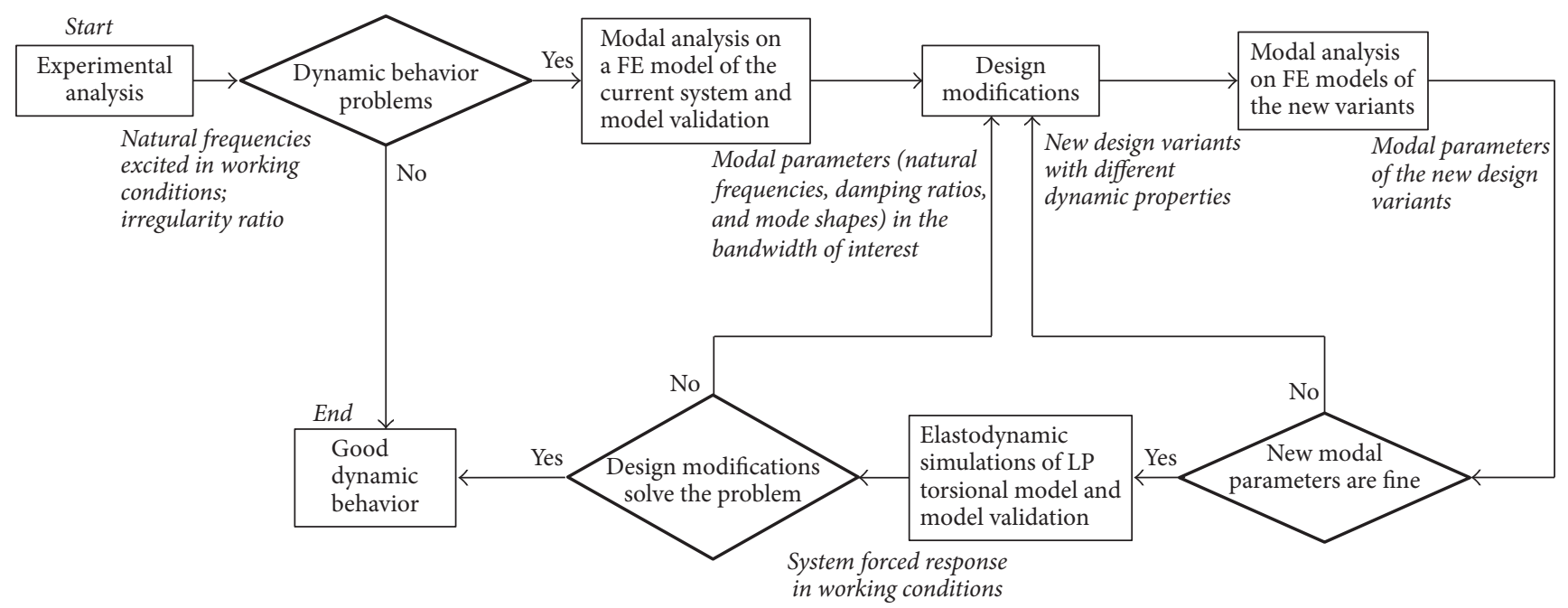

FIGURE 1: Block diagram of the proposed methodological approach.

The goal of the research is to define an effective methodology which aims at foreseeing the dynamic behavior of highly flexible couplings in real operational conditions in order to prevent a number of problems, such as high level of torsional oscillations, whirling of coupling shafts, damage of driven or driver components, and catastrophic failure of couplings or shafts. An industrial application is used as operative framework. The test rig under investigation consists of a few main components: the engine, gears, a transmission shaft, a highly flexible coupling, and a break. Despite the peculiarity of the case study described, this paper aims at proving the complexity of a complete dynamic analysis performed on this kind of mechanical system. In particular, the full understanding of the dynamic behavior requires more than one modeling approach besides an experimental activity.

The paper is organized as follows: Section 2 illustrates the adopted methodology, composed of experimental testing and numerical modeling; Section 3 reports the main results of the study and the discussion; Section 4 is finally devoted to some concluding remarks.

\section{Materials and Methods}

2.1. Methodology Outline. The proposed methodology is schematically draft in the block diagram of Figure 1 and it accounts for both numerical and experimental activities. Firstly, an experimental campaign was carried out with the aim of quantifying and characterizing the dynamic behavior of the driveline. In particular, torsional vibration measurements were performed by a coder-based technique using high-quality optical sensors and equidistantly spaced markers (zebra tape) on the rotating components. The optical sensors were mounted before and after the coupling rubber elements, at the engine-side and brake-side, respectively. As a second step, a 3D finite element (FE) model of the driveline was developed in order to estimate all the natural frequencies and mode shapes of the system in the bandwidth of interest and to evaluate which modes can negatively affect the dynamic behavior of the driveline in operational conditions. The numerical model took into account the entire driveline with particular attention to the stiffness and inertia properties of the highly flexible coupling. The FE model was experimentally validated by using data acquired during the experimental campaign mentioned above. It will be shown that the early failure of the elastic coupling is due to a resonance phenomenon of the driveline excited at particular operational conditions. Thus, the numerical model was used in order to foresee the effect of a number of design modifications proposed to reduce the negative dynamic effects. The goal was to move the natural frequencies of the driveline outside the excitation range of the engine harmonics. An iterative process entailing the proposal of new design variants and their numerical modal analyses was thus performed. Eventually, a lumped-parameter (LP) model of the entire test rig (engine, driveline, and brake) was developed in order to estimate the torsional vibration of the system in operational conditions for the different design modifications suggested by the FE analysis. In the torsional model, developed in Matlab-Simulink environment, a precise evaluation of the variable inertia properties and torque of the engine as well as the dynamic behavior of the driveline were included. The torsional LP model (of the current unaltered system) was experimentally assessed by comparison with the experimental measurements. Furthermore, the torsional model enables the evaluation of the power losses in the coupling in operating conditions, to be considered as a good feature in order to evaluate the effectiveness of design modifications.

The used methodology and the obtained results have a general meaning from a qualitative point of view. Thus, the adopted approach could be generalized to provide an effective procedure to obtain improvements in the dynamic behavior of IC engine test rig drivelines.

2.2. Case Study. A schematic of the test rig being studied is depicted in Figure 2. The test rig consists of a few main components. The crankshaft of the engine drives a transmission 


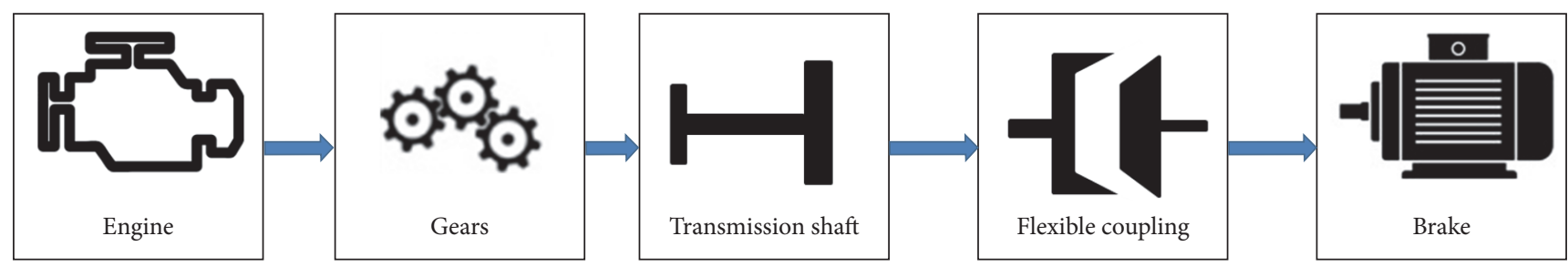

FIGURE 2: Schematic of the system under study.

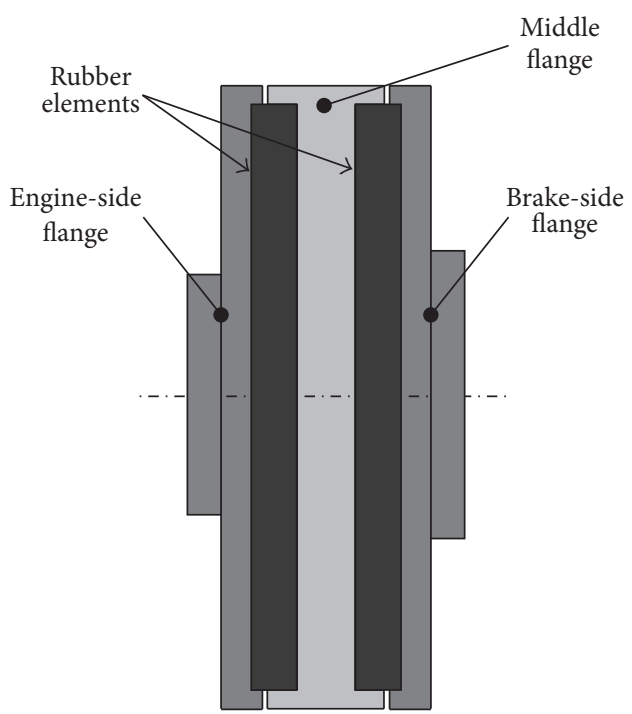

FIGURE 3: Schematic of the highly flexible coupling with rubber elements.

shaft by means of a two-stage compound gearbox with a fixed gear ratio of about $1 / 3$. The transmission shaft is connected to the electromagnetic brake through the highly flexible coupling. The coupling is composed of two rubber elements working in series and clumped to three metallic parts, namely, two outer flanges, which are joined with the transmission shaft (engine-side) and with the brake, respectively, and a middle flange that joins the rubber elements to the outer flanges (Figure 3).

2.3. Experimental Tests: Setup and Test Protocols. An experimental campaign was carried out, with the aim of characterizing the current system dynamic behavior, determining the response signature, and detecting the source of critical problems, as proposed by Troncossi et al. [9]. In particular, torsional vibration measurements were achieved by a coderbased technique using high-quality optical sensors and zebra tapes on the rotating components (Figure 4). The optical sensors were mounted on the two opposite sides of the coupling rubber elements, at the engine-side and brake-side, respectively, in order to track the torsional oscillations affecting the rubber elements. Two test campaigns were performed, corresponding to two variants of the highly flexible coupling, equipped with rubber elements having different hardness properties (and thus different stiffness). The purpose was to obtain a wide experimental database useful for the validation of numerical models of the driveline and for the coupling characterization from a dynamical standpoint. For each campaign, a brand new flexible coupling was used, avoiding any damage of a previous use. The effect of ageing of the rubber elements was not taken into account, since the time to failure due to dynamic loads was so fast to exclude any influence of the rubber age.

Torsional vibration measurements on the coupling were carried out by using two optical sensors (Optel Thevon), acquiring TTL signals from zebra tapes with line width of $2 \mathrm{~mm}$. The two sensors were equipped with two different probes (probe Optel Thevon MULTI TBYO 6M HM6X100 SURG, and probe Optel Thevon MULTI SLIT YO 6M HM6X80 SURG), both fixed to a stiff bracket. The zebra tapes were mounted in the two end-sections of the coupling, at the brake-side and engine-side (in Figure 4 the engineside tape is visible), providing the instantaneous angular speed (IAS) measurements conventionally denoted as $n_{\mathrm{bs}}$ and $n_{\mathrm{es}}$ (IAS at the brake-side and engine-side, resp.). The high number of lines per revolution (94 and 78, for the brake-side and engine-side, resp.) guaranteed a suitable angle resolution in the torsional measurements. Compensation for the data distortions due to the butt joint of the tapes was performed according to the algorithm proposed by Janssens et al. [10]. In addition, the tacho signal from a phonic wheel with 8 teeth fixed to the engine crankshaft was acquired and the corresponding IAS will be referred to as $n_{c}$ in the following. The counting rate of the optical sensors was $800 \mathrm{MHz}$ and the sampling frequency of the IAS signals was $10240 \mathrm{~Hz}$.

The system was tested in different operational conditions, namely, run-up and stationary regimes, and for different engine speeds. Run-up tests were conducted in order to find out resonant bandwidths of the system for a continuous change of engine speed. In addition, stationary tests were performed to have more precise information about the natural frequencies for a number of different constant regimes. Two test campaigns were performed, corresponding to rubber elements of the highly flexible coupling having different hardness, namely, $45 \mathrm{Sh}$ and $70 \mathrm{Sh}$ in the Shore scale, respectively. For the sake of data reliability, three different runs were performed for each test condition. The torsional oscillations of the two coupling ends were evaluated in terms of their relative velocity, denoted as $n_{\text {rel }}$ and determined as $n_{\text {rel }}=n_{\mathrm{es}}-$ $n_{\mathrm{bs}}$. Data from run-up tests were analyzed in the Time and Time-Frequency domains, whereas data measured for stationary tests were analyzed in the Time and Order domains. Velocity signals acquired in stationary tests (each one lasting $20 \mathrm{~s}$ ) were resampled with reference to the crankshaft angular 


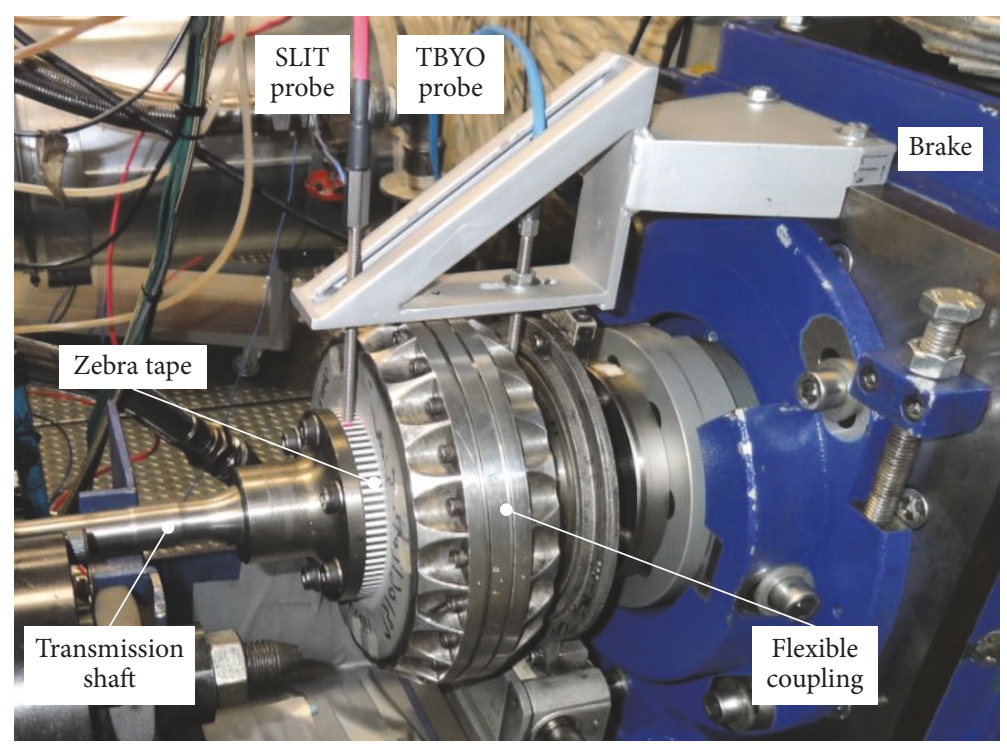

FIGURE 4: Test rig and optical sensor setup.

position [11]. Therefore, signal $n_{c}$ was taken as reference and the synchronous average [12] of all the data was performed for each thermodynamic cycle (corresponding to two crankshaft revolutions). Statistical parameters of interests (e.g., RMS values and Irregularity Ratio) were then computed on the angle-based data averages. For the frequency analysis, the Fast Fourier Transform (FFT) of the signals was computed by averaging blocks of data corresponding to 20 thermodynamic cycles. In order to associate the frequency content of the acquired signals with the excitations, the order analysis is preferred to the frequency analysis $[11,13]$. Since the major excitations of all the driveline components are due to the engine firing, an order tracking was performed by resampling data still referring to crankshaft tacho measure $n_{c}$. Timefrequency analyses of the run-up test signals, having a duration of $44 \mathrm{~s}$, were performed by computing the Short Time Fourier Transform (STFT) [14, 15], calculating the spectra in time windows of $0.1 \mathrm{~s}$ (thus providing a frequency resolution of $10 \mathrm{~Hz}$ ).

2.4. Finite Element Analysis: Model Development. A FE model was developed in order to estimate the natural frequencies and mode shapes of the driveline in the bandwidth of interest (Figure 5). The FE model accounted the transmission shaft and the highly flexible coupling. The engine and the brake were included as lumped mass elements and corresponding inertia (points (1) and (4) in Figure 5): their stiffness characteristics were neglected. Particular attention was devoted to the modeling of the transmission shaft and the rubber elements of the coupling, since they were identified as the most flexible components of the test rig. The flanges of the coupling were considered as lumped mass and corresponding inertia (points (2), (3), and (4) in Figure 5). Rigid links (namely, rigid spiders in Figure 5) were used in order to connect the lumped masses (the flanges, engine, and brake) to the $3 \mathrm{D}$ mesh of the transmission shaft and rubber elements;
Table 1 collects the features of the 3D mesh. Constraints were added in order to represent the real boundary conditions (points (A) and (B) in Figure 5). At the engine-side, the three orthogonal displacements were clamped while at the brakeside only the rotational coordinate $\theta_{z}$ was included.

A specific procedure was carried out in order to evaluate the input parameters to be included in the FE model. The coupling manufacturer provided the global torsional stiffness of the coupling, but the 3D FE model requires Young's Modulus of the rubber elements. Therefore, an iterative procedure based on a static FE model of the coupling was performed in order to estimate suitable Young's Modulus. A unitary torque was applied to the FE model of the coupling and the corresponding rotational displacement was computed. The torsional stiffness was thus calculated by means of a static analysis [15] performed with MSC.Nastran, SOL 101. Several simulations were performed by changing Young's Modulus in order to reach the torsional stiffness value provided by the manufacturer. After a few iterations, correct Young's Modulus was determined. The calculation was repeated three times in order to find Young's Modulus for three different stiffness categories (i.e., $45 \mathrm{Sh}, 60 \mathrm{Sh}$, and $70 \mathrm{Sh}$ ).

The 3D FE model requires the complete inertia tensor of the coupling flanges, whereas only the moment of inertia around the rotational axis was available from the component technical manual. In order to estimate the missing parameters, an experimental technique based on frequency response functions (FRFs) measurements was performed for the indirect measurement of the rigid body inertia properties; such a methodology is based on the well-known Inertia Restrain Method, a technique suitable for a wide range of applications when the mass distribution of components or assemblies is not known (e.g., Mucchi et al. used it in medical [16] and mechanical [17] fields). This method requires that, in the FRFs, the mass line between the highest rigid body mode and the lowest flexible body mode is rather flat. 


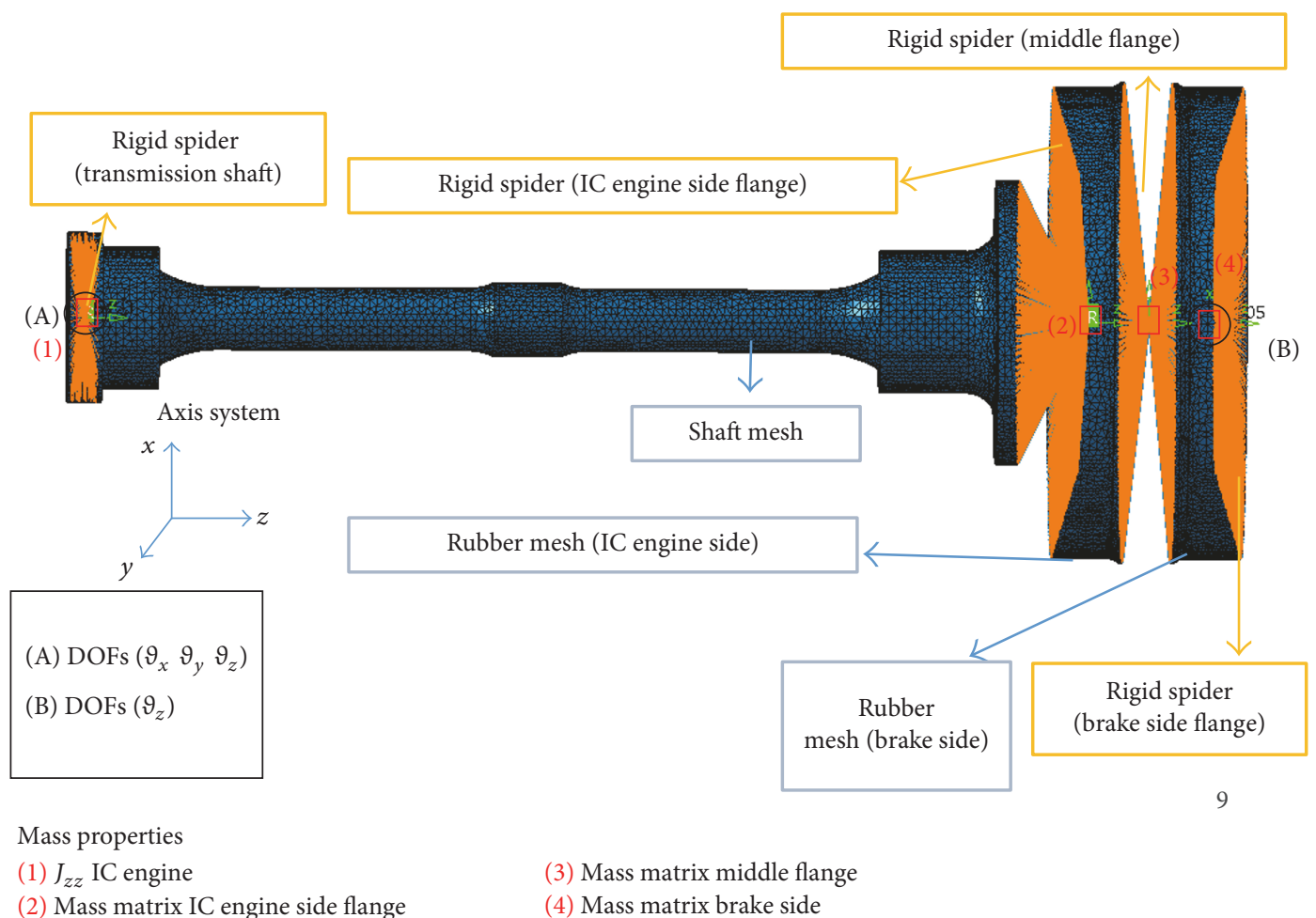

(1) $J_{z z}$ IC engine
(2) Mass matrix IC engine side flange

(4) Mass matrix brake side

Figure 5: FE model.

TABLE 1: 3D mesh features.

\begin{tabular}{lccc}
\hline & Element type & $\begin{array}{c}\text { Number of } \\
\text { nodes }\end{array}$ & $\begin{array}{c}\text { Number of } \\
\text { elements }\end{array}$ \\
\hline Transmission shaft & TETRA 4 & 23850 & 106553 \\
Rubber element & TETRA 4 & 18844 & 80412 \\
\hline
\end{tabular}

The FE model has been used in the presented research in order to simulate three design modifications (namely, MOD 1 , MOD 2, and MOD 3) conceived for moving the resonances outside the band of the engine main excitation (i.e., the band of interest). The shifting in frequency of the resonances outside the band of interest and the reduction of the number of excited modes were the tasks for improving the system dynamic behavior. At the same time, a number of design constraints had to be respected, as geometrical dimensions and final weight. The used methodology is as follows. The resonances close to the lower threshold of the bandwidth were decreased in frequency, by adding mass or reducing stiffness in specific zones, depending on the mode shape involved. The resonances close to the upper threshold were increased in frequency by reducing mass or increasing stiffness of the transmission shaft and rubber elements. In Section 3.4, the results related to the three design modifications will be outlined and then assessed by the elastodynamic analysis.

2.5. Elastodynamic Analysis: Model Development. A detailed elastodynamic model has been developed in order to capture the local modes of the coupling and to simulate the working torsional behavior, proving the effectiveness of the suggested design corrections. The elastodynamic model of the driveline was focused on the torsional dynamics only through a lumped-parameter torsional model.

The main elements of the driveline are the IC engine, the highly flexible coupling, the brake, and the shafts linking them to each other. Since the focus of the model was the coupling, it was further divided into three main parts: the two halves facing the IC engine and the brake, respectively, and the middle flange connected to the halves by means of the rubber elements. These macroelements of the driveline are indeed the same that were used in the FE model in Section 2.4. A preliminary analysis of the inertia and stiffness of the linking shafts suggested substituting them with a puretorsional spring of the same stiffness, whereas the inertia was divided between the two linked elements.

Usually, a key point of engine modeling is the loss torque due to friction associated with the piston assembly [18]. The determination of this torque is quite complex, but different types of methods have been proposed in the literature (e.g., as the ones reviewed by Richardson [19]). Performance measurement of the engine by means of power indicators was investigated by Heywood [20]. Specific experimental tests with the engine dragged at constant speed and a dynamometer recording the torque or direct friction measurement with accurate but expensive devices were discussed in [21, 22]. Rezeka and Henein [23] introduced a methodology based on the measurement of instantaneous angular velocity in the flywheel, modeling the instantaneous torque losses by means of six weighted coefficients. Later contributions detailed 


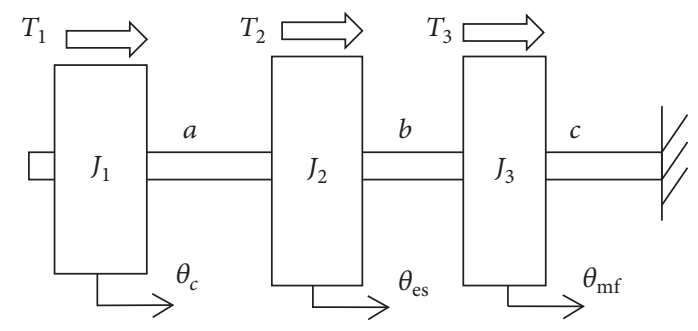

FIGURE 6: Schematic drawing of the physical model.

the computation of these coefficients in presence of local deformations [24], specific operating conditions [25], or specific configurations of the engine [26]. The six coefficients proposed by Rezeka and Henein [23] were obtained by a linear regression fitting, making the evaluation for multicylinder engines difficult. The extension to a general n-cylinder engine is due to Cruz-Peragón et al. [27] who proposed a nonlinear identification procedure of the six components of the torque losses. In this paper, an experimental computation of torque losses is out of the scope of the driveline model and it was not taken into account, but only a viscous coefficient will be used to assess the dissipative effects on the torque. The aim of the model is to provide a mathematical tool that includes the effects of parameters such as pressures, inertia, and geometry in order to adapt the model to any new configuration of the driveline.

The analysis was focused on the oscillations around steady working conditions only. In particular, the brake system was not taken into account, since the brake-side shaft is affected by negligible oscillations (see also Section 3.1, Figure 7). Thus the brake-side part of the coupling, the brake itself, and the corresponding linking shaft were considered as the fixed frame (ground). As a consequence, the mechanical system was simplified to a model with three degrees of freedom (DOFs) only, corresponding to the torsional displacements of the IC engine $\left(\theta_{c}\right)$, of the engine-side coupling part $\left(\theta_{\mathrm{es}}\right)$, and of the middle flange of the coupling $\left(\theta_{\mathrm{mf}}\right)$. Figure 6 shows a schematic drawing of the 3 DOFs model, where $J_{1}, J_{2}$, and $J_{3}$ are the inertia of the IC engine, coupling part, and middle flange, respectively. Connections $a, b$, and $c$ refer to the transmission shaft and the two rubber elements of the coupling, respectively. External torques acting on the single inertia are named as $T_{i}(i=1,2,3)$. It is worth noting that rotational coordinates $\theta_{c}$ and $\theta_{\text {es }}$ correspond to the two measured locations: the crankshaft phonic wheel and engine-side flange.

Inertia $J_{2}$ and $J_{3}$ were easy to compute since they coincide with the inertia of the single mechanical parts with respect to their center of gravity, namely, the half coupling and the middle flange. These values could be assumed from the FE analysis.

The computation of inertia $J_{1}$ was not trivial. It is the sum of different contributions of the internal components in the IC engine, some of them rotating with different angular speed. In order to reduce the complexity of the model, all main components - in terms of inertia-were reduced to the output axis of the engine, that is, the axis directly connected to the transmission shaft. The main parts of the engines considered within inertia $J_{1}$ are the alternator, the crankshaft, the gearboxes, the timing system, and the clutch.

Each linking element $(a, b$, or $c$ ) (Figure 6) was modeled as a spring and a viscous damper element working in parallel. The resulting viscoelastic torque $\left(T_{\mathrm{ve}}\right)$ can be expressed as

$$
T_{\mathrm{ve}}=k \cdot \Delta \theta+c \cdot \Delta \dot{\theta}
$$

where $k$ and $c$ are the stiffness and viscous coefficient, respectively, whereas $\Delta \theta$ and $\Delta \dot{\theta}$ are the relative rotation and relative speed of the linking element ends.

The characteristics of the transmission shaft (link $a$ ) are quite different from the rubber elements of the two coupling halves (links $b$ and $c$ ).

The dynamic behavior of rubber could be very complex, due to nonlinearity of the material response. Different models have been proposed in the literature. Qi and Boyce [28] proposed a constitutive model capturing the major features of the stress-strain behavior of thermoplastic polyurethanes, including nonlinear hyperelastic behavior, time dependence, hysteresis, and softening. Wei and Kukureka [29] proposed a resonance technique for determining the stiffness and damping properties of a composite or composite structure. Chen et al. [30] suggested a frequency-domain method for estimating the mass, stiffness, and damping matrices of the model of a structure, based on the extraction of normal modes from the complex modes, by means of a transformation matrix. Cruz-Peragón et al. [8] developed a methodology to identify the coupling characteristics to validate dynamic models of engine assemblies with flexible coupling. The method is based on static and dynamic tests, nonlinear models, and techniques for parameter identification. These methods are powerful tools for the characterization of the material and its implementation in nonlinear models of mechanical systems. In this paper the focus of the lumped-parameter model is on the frequency response of the flexible coupling, rather than a characterization of the rubber material. Since natural frequencies are not defined for nonlinear models, a linear model of the rubber coupling has been considered.

While the viscous damper model is correct for the steel material (in the elastic domain), for the rubber material an equivalent viscous coefficient was computed starting from a hysteretic damping model [31]. The resulting viscous coefficient is

$$
c=\frac{k \cdot \gamma}{2 \pi \cdot \omega}
$$

where $\gamma$ is the relative damping value of the rubber and $\omega$ is the angular speed of the hysteretic loop. In this model $\omega$ is the speed of the IC engine cycle.

The only nonzero external torque is $T_{1}$, acting on inertia $J_{1}\left(T_{2}=T_{3}=0\right) . T_{1}$ is the result of the combustion cycles of the engine in each cylinder. The torque contribution of each cylinder comes from the expansion phase in the IC cycle and the inertia momentum of each part of the crankslide mechanism. With reference to the nomenclature in "Nomenclature," the contribution of the four cylinders to the 
external torque is properly combined considering the relative phases:

$$
\begin{aligned}
T_{1} & =\sum_{i=1}^{4}\left[P_{i} \cdot \pi \cdot\left(\frac{d}{2}\right)^{2} \cdot r_{v i}-\left(m_{P}+m_{A}-\frac{J_{0}}{l^{2}}\right)\right. \\
& \left.\cdot\left(\dot{\phi}_{i}^{2} \cdot r_{a i}+\ddot{\phi}_{i} \cdot r_{v i}\right) \cdot r_{v i}\right],
\end{aligned}
$$

where

$$
\begin{aligned}
m_{A} & =m_{b} \cdot \frac{l_{b}}{l} \\
J_{0} & =J_{b}-m_{b} l_{b} l_{a} \\
r_{v i} & =r\left(\sin \phi_{i}+\frac{r}{2 l} \sin 2 \phi_{i}\right) \\
r_{a i} & =r\left(\cos \phi_{i}+\frac{r}{l} \cos 2 \phi_{i}\right) .
\end{aligned}
$$

It should be noted that the pressures in the combustion chambers $\left(P_{i}\right)$ were estimated by experimental measurements previously performed by the engine manufacturer.

A detailed description of the formula in (3) and (4) can be found in several references about the theory of machines and mechanisms, for example, in the book of Uicker et al. [32]. The quantities in (3) and (4) without subscript index were supposed to be the same for all the cylinders, whereas the other ones differed along the combustion phase $\left(\varphi_{i}\right)$ of each cylinder with respect to a certain reference (e.g., the angular displacement $\theta_{c}^{*}$ of the inertia $J_{1}$ ):

$$
\phi_{i}=\theta_{c}^{*}+\varphi_{i} \quad i=1, \ldots, 4 .
$$

The superscript asterisk recalls that the angular displacement in (5) should be the absolute displacement, not just the oscillation of inertia $J_{1}$ around the equilibrium configuration.

The equations of torsional motion of the three DOFs system were arranged in matrix form:

$$
\begin{gathered}
{\left[\begin{array}{ccc}
J_{1} & 0 & 0 \\
0 & J_{2} & 0 \\
0 & 0 & J_{3}
\end{array}\right]\left[\begin{array}{c}
\ddot{\theta}_{c} \\
\ddot{\theta}_{\mathrm{es}} \\
\ddot{\theta}_{\mathrm{mf}}
\end{array}\right]+\left[\begin{array}{ccc}
c_{1} & -c_{1} & 0 \\
-c_{1} & c_{1}+c_{2} & -c_{2} \\
0 & -c_{2} & c_{2}+c_{3}
\end{array}\right]\left[\begin{array}{c}
\dot{\theta}_{c} \\
\dot{\theta}_{\mathrm{es}} \\
\dot{\theta}_{\mathrm{mf}}
\end{array}\right]} \\
+\left[\begin{array}{ccc}
k_{1} & -k_{1} & 0 \\
-k_{1} & k_{1}+k_{2} & -k_{2} \\
0 & -k_{2} & k_{2}+k_{3}
\end{array}\right]\left[\begin{array}{c}
\theta_{c} \\
\theta_{\mathrm{es}} \\
\theta_{\mathrm{mf}}
\end{array}\right]=\left[\begin{array}{c}
T_{1} \\
0 \\
0
\end{array}\right],
\end{gathered}
$$

where $c_{2}$ and $c_{3}$ come from (2) and $T_{1}$ comes from (3). Equations were numerically integrated in Simulink environment. Other details about Simulink implementation can be found in [33].

\section{Results}

3.1. Experimental Run-Up Test Results. Hereafter, the results relative to the current system (with $45 \mathrm{Sh}$ rubber elements of the coupling) will be firstly discussed, with the aim of highlighting the dynamic effects that likely led to the early collapse of the rubber elements. Then, the main data resulting from the substitution of the $45 \mathrm{Sh}$ rubber elements with harder ones $(70 \mathrm{Sh})$ will be shown and compared with the previous ones. Due to confidentiality agreement with the industrial partner, no data related to the shaft velocities can be explicitly reported. Therefore, data relative to run-up tests will be shown as normalized to $n_{c}$ maximum value.

A preliminary comparison among the data acquired in the three different runs confirmed the extreme repeatability of results, being negligible any difference. The following results correspond to the second runs performed (for both the $45 \mathrm{Sh}$ and $70 \mathrm{Sh}$ rubber element cases). In Figure 7 the time series of the acquired IAS are plotted as scaled to the maximum value of the crankshaft velocity $n_{c}$. In the case of $45 \mathrm{Sh}$ rubber elements (Figure 7(a)), the oscillation of the velocity $n_{\mathrm{es}}$ of the engine-side flange of the coupling is much higher and more irregular than the brake-side one $\left(n_{\mathrm{bs}}\right)$, which exhibits a very smooth trend (thus meaning that the brakeside shaft is affected by negligible oscillations). In particular, $n_{\text {es }}$ is subjected to a very high increment after the sixteenth second of the run. The analysis of this oscillation is the main tool to understand the dynamic phenomenon underlying the system behavior. To this aim, the analysis was focused on the relative velocity between the two coupling ends, $n_{\text {rel }}$, which was analyzed in the Time-Frequency domain to highlight the presence of resonant bands of the system. Figure 8 presents the STFT in the frequency range between $0 \mathrm{~Hz}$ and $f_{\max }$ (not made explicit for confidential reason). In Figure 8(a) it can be noted that the frequency content of $n_{\text {rel }}$ is dominated by order 1 of the crankshaft rotation and-to a smaller extent-by orders $0.5,1.5$, and 2 , being order 0.5 associated with the engine thermodynamic cycle. Starting from the sixteenth second of the run-up, the amplitude of $n_{\text {rel }}$ significantly increases with a frequency content firstly associated with the crankshaft order 0.5 (16-18 s) and then dominated by order 1 for a long time interval (18-30 s). Two natural frequencies, whose value is here symbolically reported as $f_{1}$ and $f_{2}$, are excited in these two phases, being $f_{2}$ widespread in a large bandwidth. It could be noted that the second one is excited also by the crankshaft order 1.5, with lower energy, in the interval 8-12 s. Other possible higher resonances, $f_{3}$ and $f_{4}$, are slightly excited by the crankshaft orders 1.5 and 2 at about $18-20 \mathrm{~s}$, but with low energy. The RMS value of $n_{\text {rel }}$ computed for the entire duration of the run-up is about $156 \mathrm{rpm}$.

The further test campaign carried out with the coupling carrying on the 70 Sh rubber elements led to significantly different results (Figure 7(b)). As expected, the stiffening effect of the harder rubber increased the system natural frequencies and brought about benefits on the dynamic behavior of the driveline: the oscillations of speed $n_{\mathrm{es}}$ of the engine-side flange were indeed significantly smaller than in the previous campaign, whereas $n_{\mathrm{bs}}$ was basically the same (Figure 7). As a consequence, relative velocity $n_{\text {rel }}$ was significantly lower, as it can be seen in Figure 8(b), where the full scales of time plot and colormap are the same as in Figure 8(a) in order to highlight the important reduction. The RMS value of $n_{\text {rel }}$ computed for the entire duration of the run-up was $74 \mathrm{rpm}$, 
that is, less than the half of the previous campaign value. Some dynamic effects were still present, though resulting in speed oscillations smaller than in the case of the $45 \mathrm{Sh}$ rubber elements. In particular, the signal was dominated by crankshaft order 1 , which excited natural frequency $f_{1}{ }^{*}$ at the time interval 10-14 s, corresponding to a velocity that was about $50 \%$ of the final run-up velocity. Moreover, at the very beginning of the run-up (for a velocity of about $30 \%$ of the maximum value), order 0.5 seemed to excite resonance $f_{0}{ }^{*}$. The asterisk symbol is introduced in order to have explicit reference to the natural frequency values for the 70 Sh coupling, which are obviously different with respect to the 45 Sh case.

3.2. Experimental Stationary Test Results. Stationary tests at different velocities were performed. The results of a limited, significant selection are presented and discussed in the paper and the corresponding runs are conventionally referred to as Regime A, Regime B,..., Regime F (for confidentiality reasons), where Regime $\mathrm{A}$ and Regime $\mathrm{F}$ are about $50 \%$ and $94 \%$, respectively, of the maximum speed achieved in the run-up tests and that will be kept as reference in the paper. After resampling and synchronously averaging the velocity signals based on the rotation of the crankshaft, the angle and order analyses as well as the time statistics are available. In particular, a more accurate estimation of the system natural frequencies with respect to the run-up results was achieved.

Starting again from the analysis of the $45 \mathrm{Sh}$ results, Figures 9(a) and 9(c) report the crankshaft-angle-based trend of $n_{\text {rel }}$ over five thermodynamic cycles and its orderbased spectral analysis for Regime C (limited to the first two crankshaft orders), which is about $66 \%$ of the run-up maximum velocity (i.e., the velocity achieved at the twentieth second of the run-up in Figure 7). The consistency with the time-frequency analysis of the run-up tests appears to be evident: $n_{\text {rel }}$ signal is indeed dominated by crankshaft orders 1 and 2 , partly exciting the resonant frequency $f_{2}$ and $f_{4}$, and at a lower extent by orders 0.5 and 1.5, which excite resonant frequencies $f_{1}$ and $f_{3}$, respectively. A minor contribution of the transmission shaft orders is also somehow appreciable (corresponding to multiples of about one-third of the crankshaft order 1). It is worth noticing the presence of a low frequency resonance $f_{0}$ corresponding at about 0.1 crankshaft order. This natural frequency was excited in each stationary regime, but at a negligible level since the excitation coming from the engine does not contain the corresponding harmonics content. Therefore, the excitation of such a resonance was likely due to low frequency mechanical noise present in each test.

Table 2 reports the most significant statistical values of $n_{\text {rel }}$ computed for the stationary tests, that is, the RMS value and the "Irregularity Ratio" (IR) between the $n_{\text {rel }}$ peak-to-peak amplitude and the mean value of the transmission shaft velocity. Since the results obtained from the three runs acquired for each condition proved very repeatable, data are reported referring to one run only (the second one acquired). The maximum and the mean absolute values of the differences among the runs in the computation of IR are $+0.8 \%$ (Regime B) and $0.29 \%$, respectively. Consistently with the timefrequency analysis, Regime $\mathrm{C}$ represents the most critical
TABLE 2: Statistical parameters of $n_{\text {rel }}$ computed for the stationary tests.

\begin{tabular}{lcccc}
\hline \multirow{2}{*}{$n_{\text {rel }}$} & \multicolumn{2}{c}{45 Sh } & \multicolumn{2}{c}{70 Sh } \\
& RMS [rpm] & IR [\%] & RMS [rpm] & IR [\%] \\
\hline Regime A & 115 & 15.4 & 125 & 17.0 \\
Regime B & 183 & 21.2 & 63 & 8.0 \\
Regime C & 245 & 25.2 & 42 & 5.2 \\
Regime D & 236 & 18.8 & 34 & 4.0 \\
Regime E & 173 & 13.2 & 31 & 3.2 \\
Regime F & 94 & 6.8 & 72 & 5.2 \\
\hline
\end{tabular}

regime in terms of oscillations that the rubber elements are subjected to (in the case of the $45 \mathrm{Sh}$ rubber elements).

From the analysis of all the data retrieved from both runup and stationary tests performed on the current test rig (with $45 \mathrm{Sh}$ rubber elements), it can be concluded that five natural frequencies were likely present in the bandwidth $0-f_{\max }$. In particular,

(i) $f_{0}$ was never significantly excited;

(ii) $f_{1}$ was excited by crankshaft order 0.5 at a velocity corresponding about to Regime B;

(iii) $f_{2}$ was excited by both orders 1 and 1.5 depending on the crankshaft speed;

(iv) the medium-high natural frequencies $f_{3}$ and $f_{4}$ were excited with low energy by crankshaft orders 1.5 and 2.

The most important contribution to the coupling relative oscillations was provided by natural frequency $f_{2}$, which is spread in quite a large band. Efforts to improve the dynamic response of the highly flexible coupling (and the entire driveline as a consequence) should be thus focused at moving these resonant bands away from the bandwidth that can be excited by crankshaft orders 0.5 to 2 (while not introducing, at the same time, other resonances in this bandwidth).

Table 2 also reports the statistical parameters computed for the stationary tests (second run) conducted with the $70 \mathrm{Sh}$ rubber elements. The maximum and the mean absolute values of the differences among the runs in the computation of IR are $-0.6 \%$ (Regime A) and $0.21 \%$, respectively. The global decrement of the oscillation amplitudes appears evident for Regimes B to F, for which the current coupling presented its critical response. In spite of this great improvement, the new coupling response was slightly worse for Regime A. Figures 9(b) and 9(d) report the crankshaft-angle-based trend of $n_{\text {rel }}$ and its order-based spectral analysis for Regime $\mathrm{C}$, for a direct comparison with the analogous analysis presented for the $45 \mathrm{Sh}$ case. Figure 10 reports the same quantities relative to Regime A at $70 \mathrm{Sh}$. In these figures the resonant frequencies are appreciable as quite widespread hills among the narrowband peaks corresponding to the crankshaft and transmission shaft orders. Since the harder rubber entails a higher stiffness of the coupling, it is reasonable to conclude that natural frequencies $f_{0}$ and $f_{1}$ of the 45 Sh rubber coupling (corresponding, resp., about to 0.10 and 0.45 crankshaft orders, for Regime C, Figure $9(\mathrm{c})$ ) moved to the higher values $f_{0}{ }^{*}$ and $f_{1}{ }^{*}$ for 

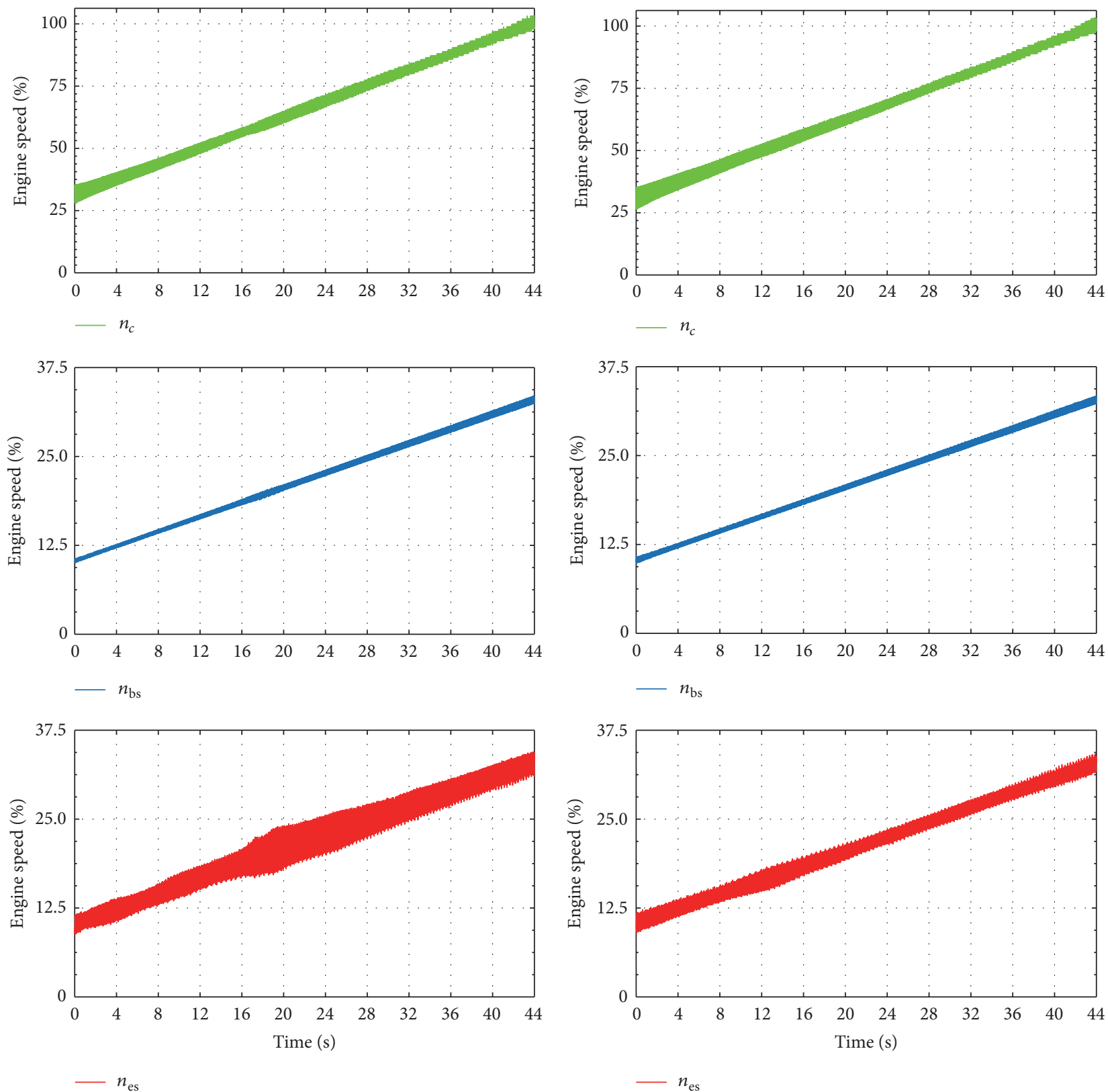

(a)

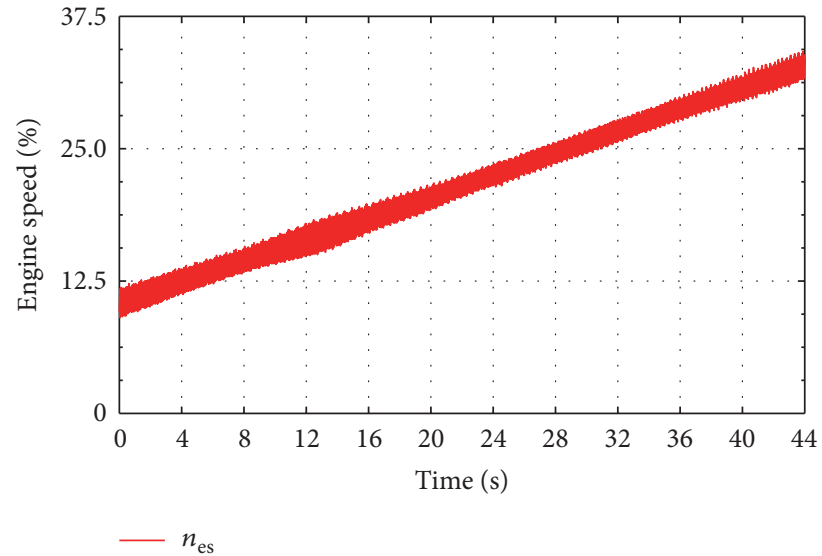

(b)

FIGURE 7: Time data of the normalized velocity signals acquired during a run-up test: (a) 45 Sh rubber elements and (b) 70 Sh rubber elements.

the 70 Sh rubber coupling (corresponding about to 0.20 and 0.77 orders for Regime C, Figure 9(d), and to 0.25 and 0.96 for Regime A, Figure 10(b)). No other natural frequencies seemed to be excited for the 70 Sh rubber coupling for any regime achieved in the run-up and in the stationary tests.

In order to analyze if the stiffening effect of the harder rubber induced secondary effects in other parts of the driveline, the IAS $n_{c}$ of the crankshaft is compared both for the run-up and stationary tests. The colormaps in Figure 11 (sharing the same full scale) report the comparison between the STFTs of $n_{c}$, whereas Table 3 reports the RMS values of the crankshaft velocity oscillations, $\Delta n_{c}$, and the IR values computed for the seven stationary tests. The data analysis reveals that the oscillations of $n_{c}$ were slightly higher at lowmedium regimes in the case of the $70 \mathrm{Sh}$ rubber, but to a negligible extent so that no problematic operations were induced on the whole system.

It is worth recalling that the performed experimental analysis did not permit determining the vibration modes associated with the mentioned natural frequencies. In other words, it is not possible to state that only torsional modes were excited, since it cannot be excluded that a flexural mode of the transmission shaft could induce coupled oscillations in the IAS. 

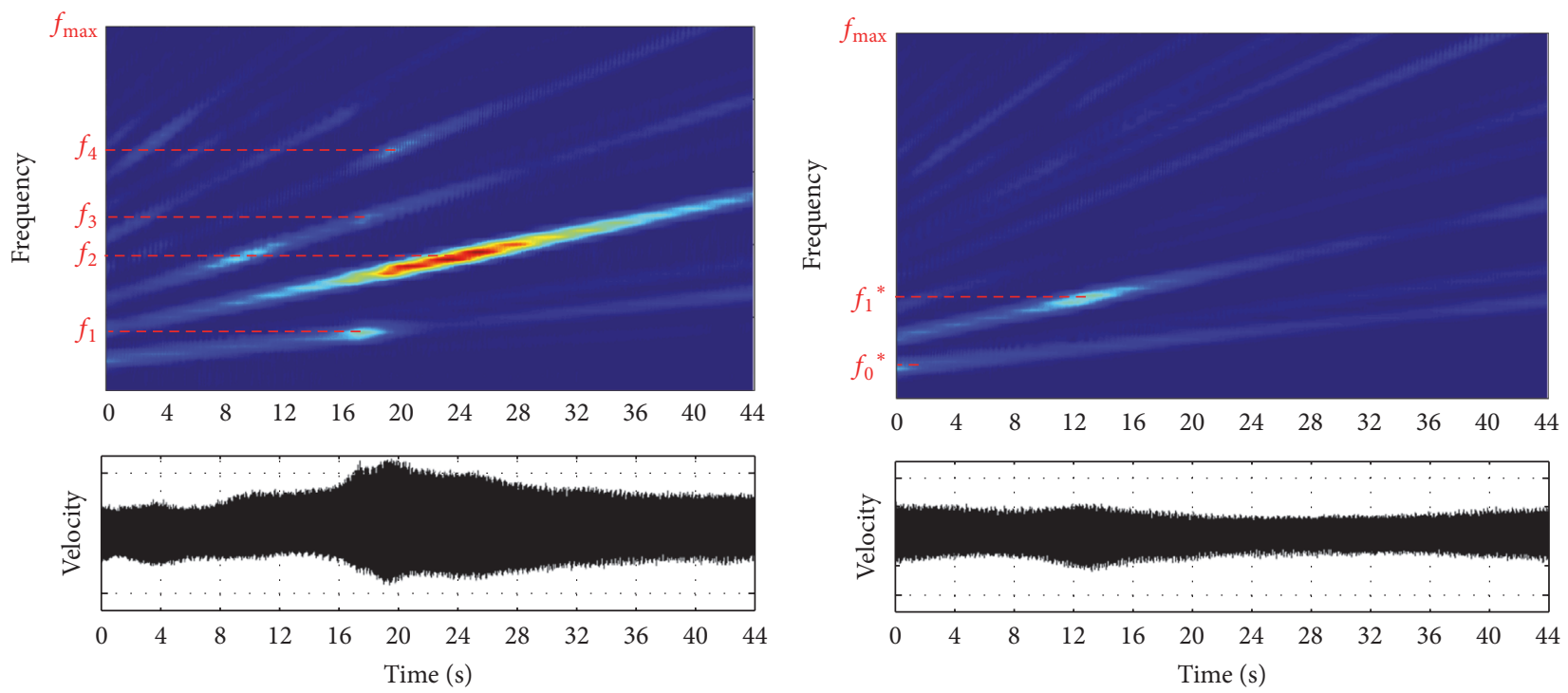

(a)

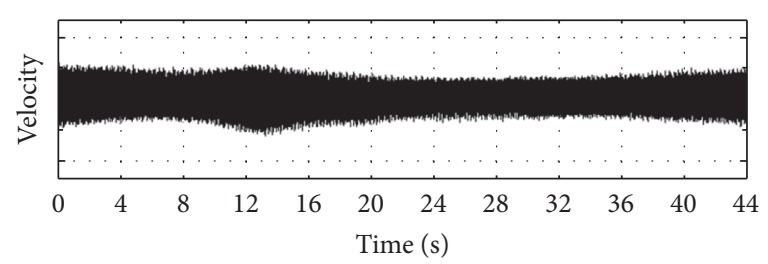

(b)

FIGURE 8: Time-frequency analysis of the relative velocity $n_{\text {rel }}$ computed for a run-up test: (a) 45 Sh rubber elements and (b) 70 Sh rubber elements.

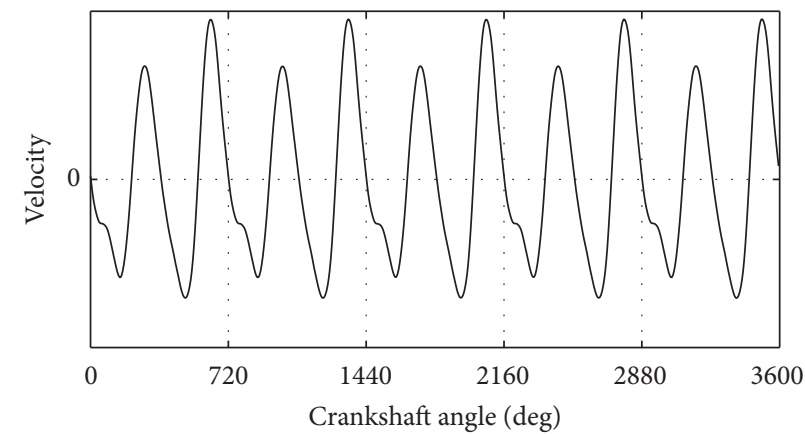

(a)

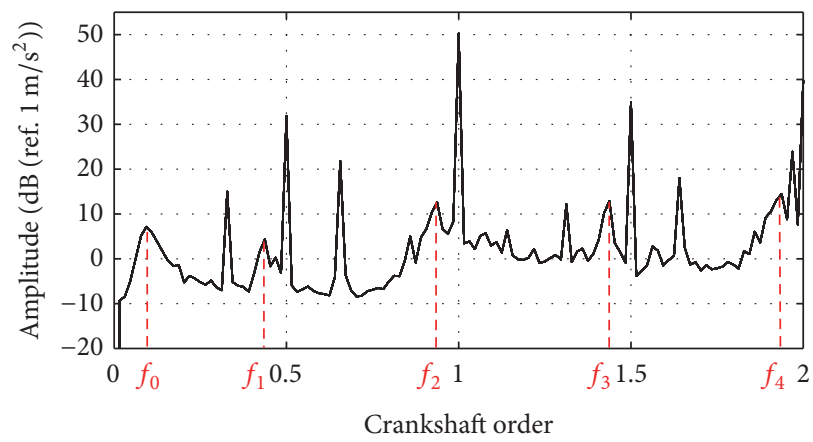

(c)

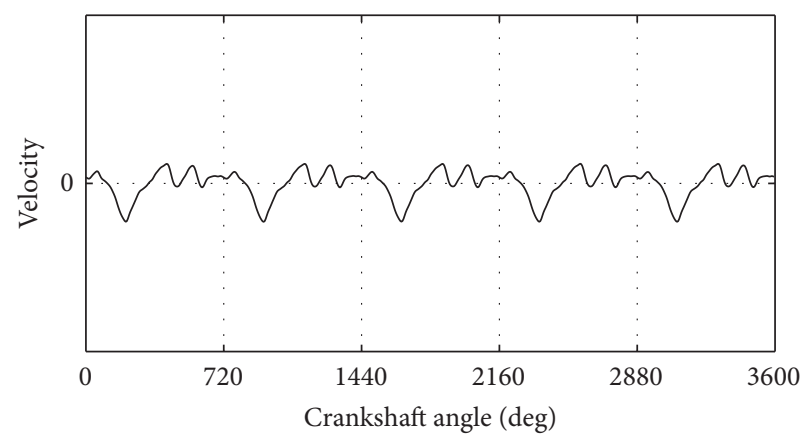

(b)

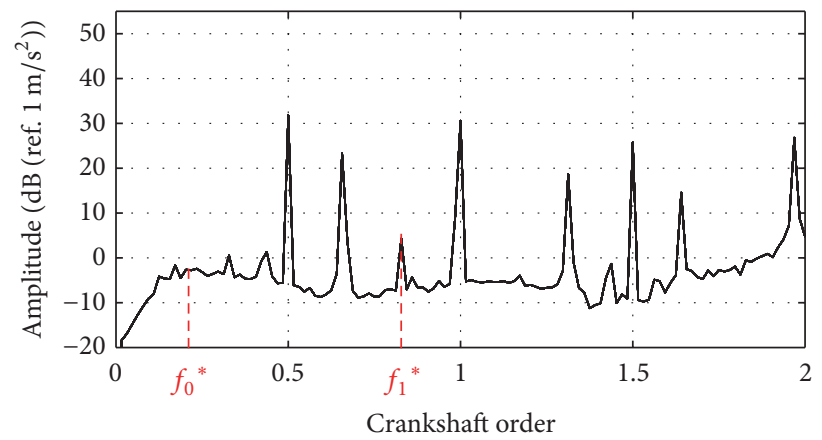

(d)

Figure 9: Crankshaft-angle-based trend (reported over five thermodynamic cycles) and order-based spectral analysis of $n_{\text {rel }}$ for Regime C: (a, c) 45 Sh and (b, d) 70 Sh.

3.3. Finite Element Analysis: Model Validation. Table 4 collects the elastic properties of the rubber element estimated by the FE static analysis described in Section 2.4, the inertia properties estimated by the experimental procedure, and the moment of inertia of the flanges around the rotational axis provided by the manufacturer. These data have been used as input data in the numerical modal analysis (Sol 103 in MSC.Nastran) presented in Section 2.4.

The simulation results obtained through a numerical modal analysis (Sol 103 in MSC.Nastran) regarding 45 Sh and 70 Sh rubber configurations were compared with measurements (Sections 3.1 and 3.2). The experimental STFTs with 


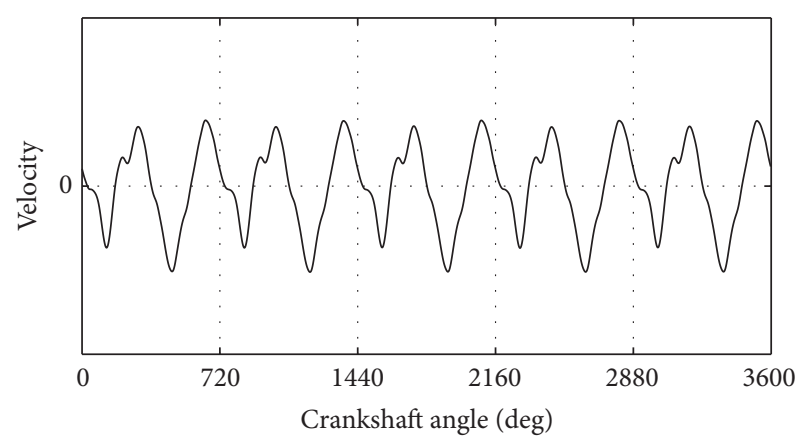

(a)

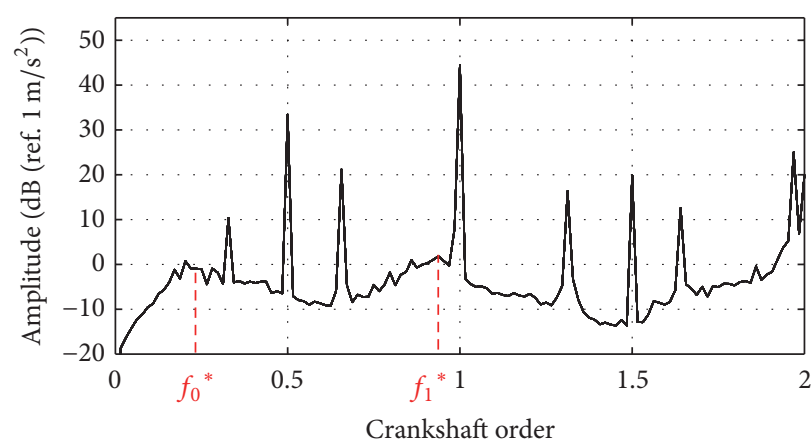

(b)

FIGURE 10: Crankshaft-angle-based trend and order-based spectral analysis of $n_{\text {rel }}$ for Regime A of the 70 Sh tests.
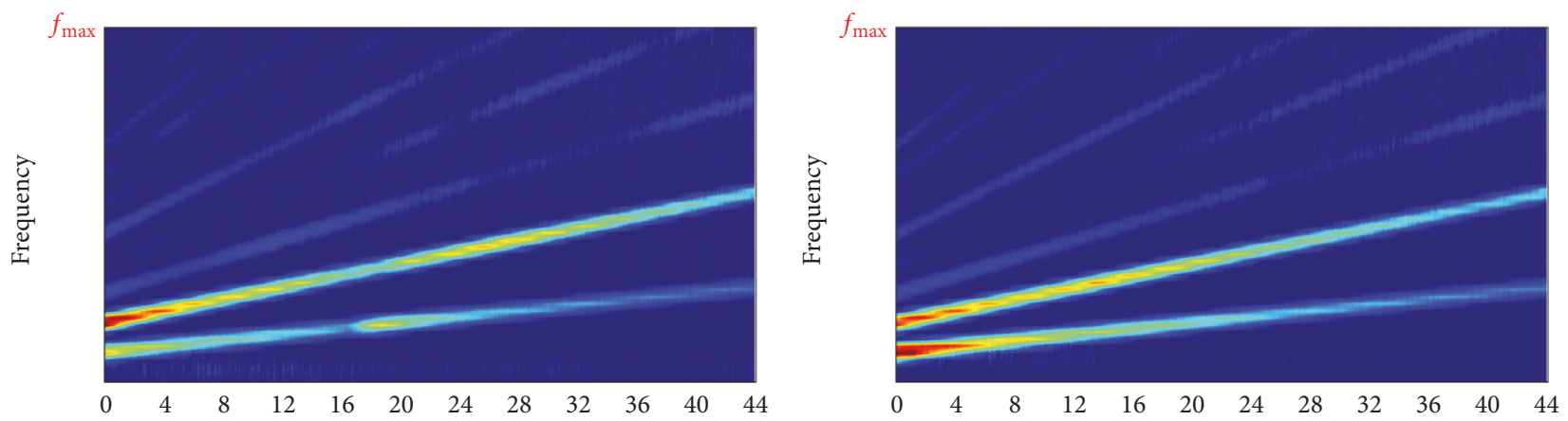

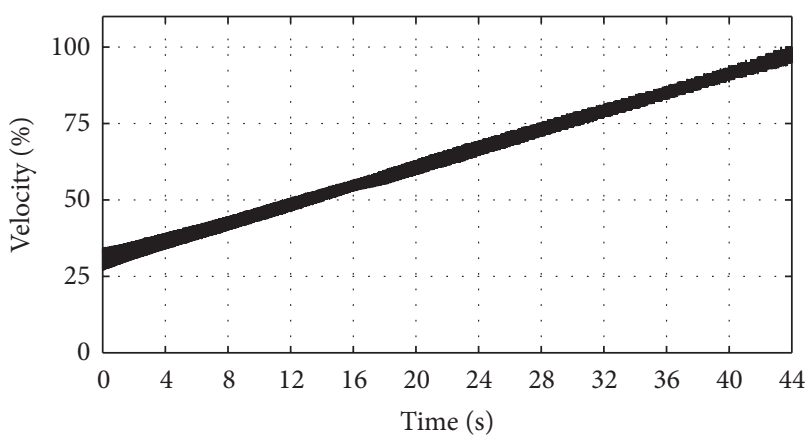

(a)

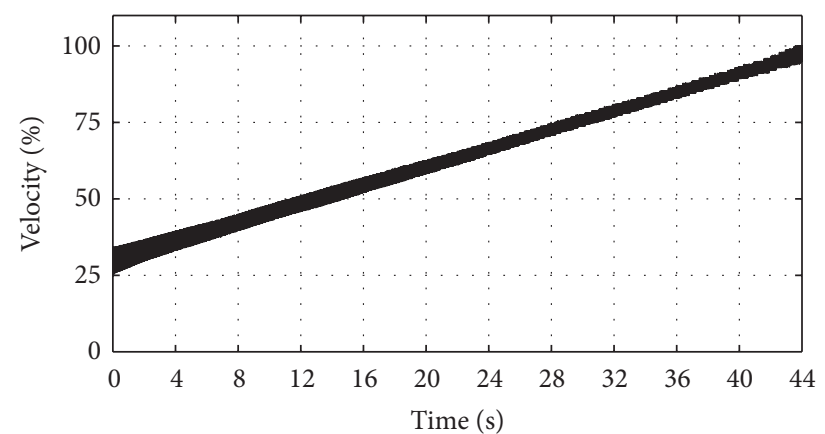

(b)

FIGURE 11: Time-frequency analysis of the crankshaft velocity $n_{c}$ computed for a run-up test: (a) 45 Sh rubber elements and (b) 70 Sh rubber elements.

the two rubber configurations clearly show a few resonance regions in the frequency range $0-f_{\max }$ that are collected in Tables 5(a) and 6(a). Tables 5(b) and 6(b) collect the natural frequencies estimated by the FE analysis. Regarding the $45 \mathrm{Sh}$ rubber configuration, four numerical modes are very close to the resonance regions detected by the experimental STFT. The first matching concerns the first torsional mode of the driveline, where the elements in the zone between the engine-side and the middle flange move out of phase with respect to the brake ( $3 \%$ versus $2.9 \%$ of $f_{\max }$ ). The second matching regards the second torsional mode, which is characterized by a high amplitude displacement of the middle flange ( $14 \%-16 \%$ versus $14.9 \%$ of $\left.f_{\max }\right)$. Finally, the last two experimental frequencies $(32 \%-38 \%$ and $68 \%$ of $\left.f_{\max }\right)$ correspond, respectively, to the 5 th $\left(36.8 \%\right.$ of $\left.f_{\max }\right)$ and the 8 th $\left(69.7 \%\right.$ of $\left.f_{\max }\right)$ numerical modes. In particular, the 5 th numerical mode is a double mode (two roots at same frequency due to symmetry) and involved the rotation of the middle flange around axes $y$ and $x$. This is the mode mainly excited in operational conditions. The remaining modes detected in the FE analysis are not present in the experimental map, since they are not excited by the engine harmonics in operational conditions due to their particular shape. Similar considerations can be done for the $70 \mathrm{Sh}$ rubber configuration (Table 6). The comparison between the experimental and the numerical results shows that the mode which determines the highest peaks in the experimental maps regards the third local mode of the coupling. Thus, attention should be paid in order to move this mode far away from the excitation harmonics. 
TABLE 3: Crankshaft velocity oscillations RMS value, $\Delta n_{c}$, and Irregularity Ratio, IR, computed for the stationary tests performed with the two highly flexible couplings.

\begin{tabular}{lcccc}
\hline$n_{c}$ & \multicolumn{3}{c}{$45 \mathrm{Sh}$} & \multicolumn{2}{c}{ Sh } \\
& $\begin{array}{c}\Delta n_{c \_ \text {RMS }} \\
{[\mathrm{rpm}]}\end{array}$ & $\begin{array}{c}\text { IR } \\
{[\%]}\end{array}$ & $\begin{array}{c}\Delta n_{c \_ \text {RMS }} \\
{[\mathrm{rpm}]}\end{array}$ & $\begin{array}{c}\text { IR } \\
{[\%]}\end{array}$ \\
\hline Regime A & 177 & 7.8 & 239 & 10.6 \\
Regime B & 153 & 6.0 & 205 & 7.8 \\
Regime C & 193 & 7.0 & 182 & 6.2 \\
Regime D & 187 & 6.4 & 159 & 4.8 \\
Regime E & 176 & 5.0 & 144 & 4.0 \\
Regime F & 144 & 3.6 & 139 & 3.2 \\
\hline
\end{tabular}

TABLE 4: Input data for FE analysis.

\begin{tabular}{lc}
\hline Rubber properties & Value \\
\hline Rubber density (45-60-75 Shore) & $1000 \mathrm{~kg} / \mathrm{m}^{3}$ \\
Poisson's ratio & 0.49 \\
Young's Modulus rubber 45 Shore $\left(60^{\circ} \mathrm{C}\right)$ & $1.32 E+06 \mathrm{~N} / \mathrm{m}^{2}$ \\
Young's Modulus rubber 60 Shore $\left(60^{\circ} \mathrm{C}\right)$ & $3.11 E+06 \mathrm{~N} / \mathrm{m}^{2}$ \\
Young's Modulus rubber 70 Shore $\left(60^{\circ} \mathrm{C}\right)$ & $4.72 E+06 \mathrm{~N} / \mathrm{m}^{2}$ \\
\hline Coupling inertial properties & $5.68^{*} \mathrm{~kg}$ \\
\hline Mass & $0.01337^{* *} \mathrm{kgm}{ }^{2}$ \\
$J_{x x}$ & $0.01337^{* *} \mathrm{kgm}^{2}$ \\
$J_{y y}$ & $0.01961^{* *} \mathrm{kgm}^{2}$ \\
$J_{z z}$ & ${ }^{*}$ Data from catalogue of manufacturer. ${ }^{* *}$ Data from experiments.
\end{tabular}

TABLE 5: 45 Sh configuration. (a) List of experimental resonances. (b) List of numerical (FE) natural frequencies, in percentage of $f_{\max }$.

(a) $45 \mathrm{Sh}$ : experimental frequencies

\begin{tabular}{lcc}
\hline Mode 1 & & $\sim 3 \%$ \\
Mode 2 & $14 \%-16 \%$ \\
Mode 3 & & $32 \%-38 \%$ \\
Mode 4 & (b) 45 Sh: numerical results & $\sim 68 \%$ \\
\hline & $2.9 \%$ (1st global torsional mode [1st TG]) \\
\hline Mode 1 & $7 \%$ (1st local mode of coupling [1st LC]) \\
Mode 2 & $14.9 \%$ (2nd global torsional mode [2nd TG]) \\
Mode 3 & $19.7 \%$ (2nd local mode of coupling [2nd LC]) \\
Mode 4 & $36.8 \%$ (3rd local mode of coupling [3rd LC]) \\
Mode 5 & $44 \%$ (3rd global torsional mode [3rd TG]) \\
Mode 6 & $50.6 \%$ (1st axial mode middle flange [1st AF]) \\
Mode 7 & $69.7 \%$ (1st bending mode output shaft [1st BS]) \\
Mode 8 &
\end{tabular}

3.4. Finite Element Analysis: Dynamic Behavior Improvement. Experimental results clearly showed that, within the frequency band of interest, that is, $18 \%-60 \%$ of $f_{\max }$-where the main excitation harmonics due to the engine lie-resonances occur. Moreover, FE simulations permitted defining the corresponding mode shapes of such resonances (Section 3.3).
TABLE 6: 70 Sh configuration. (a) List of experimental resonances. (b) List of numerical (FE) natural frequencies, in percentage of $f_{\max }$.

(a) $70 \mathrm{Sh}$ : experimental frequencies

\begin{tabular}{lc}
\hline Mode 1 & $6 \%-8 \%$ \\
Mode 2 & $24 \%-30 \%$ \\
\hline
\end{tabular}

(b) 70 Sh: numerical results

\begin{tabular}{cc}
\hline Mode 1 & $5.1 \%[1 \mathrm{st} \mathrm{TG}]$ \\
Mode 2 & $13 \%[1 \mathrm{st} \mathrm{LC}]$ \\
Mode 3 & $25.7 \%[2 \mathrm{nd} \mathrm{TG}]$ \\
Mode 4 & $37.2 \%[2 \mathrm{nd} \mathrm{LC}]$ \\
Mode 5 & $60.2 \%[3 \mathrm{rd} \mathrm{TG}]$ \\
Mode 6 & $60.4 \%[3 \mathrm{rd} \mathrm{LC}]$ \\
Mode 7 & $83.6 \%[1 \mathrm{st} \mathrm{AF}]$ \\
Mode 8 & $85.1 \%[1 \mathrm{st} \mathrm{BS}]$ \\
\hline
\end{tabular}

This section presents the results of the three design modifications MOD 1, MOD 2, and MOD 3 proposed to move the resonances outside the band of interest.

The first design modification (MOD 1) regarded the 45 Sh rubber element configuration; MOD 1 addressed an increased weight of the middle flange that reduced at $17.8 \%$ the frequency of the third local mode of the coupling. Furthermore, the steel transmission shaft was replaced by a stiffer titanium shaft keeping the third torsional mode outside the band of interest. Eventually, a flywheel was introduced on the engine shaft in order to keep the second torsional frequency at low frequency. The comparison between Tables 5 and 7 (a) shows that targets are successfully reached, but MOD 1 leads to a rather heavy design, which could entail problems at high-speed conditions. It is worth noting that Mode 6 remains in the band of interest after the modification, but due to its particular shape it is not excited in operational conditions.

The second design modification (MOD 2) concerned the $70 \mathrm{Sh}$ rubber element configuration, where the middle flange was lightened and a flywheel on the engine shaft and a stiffer transmission shaft were placed. Table 7 (b) collects the resulting natural frequencies. The 2 nd torsional mode (2nd TG) still remains in the band of interest: in spite of this drawback, the global benefits of this modification will be illustrated in Section 3.6. Moreover, as stated in Section 3.2, the second local mode of the coupling (2nd LC) is not excited in operational conditions.

The last design modification (MOD 3) took into consideration the use of rubber elements with 60 Sh hardness. The standard middle flange was lightened by milling some parts and by replacing steel screws with titanium ones. The transmission shaft was included in titanium and a flywheel was mounted on the engine shaft. Table 7 (c) collects the natural frequencies. The third local mode of the coupling is above the band of interest threshold, whereas the second torsional mode is still inside the band of interest. However, the $60 \mathrm{Sh}$ rubber has the highest relative damping value; thus the vibration amplitude at this resonance is expected to be reduced with respect to $70 \mathrm{Sh}$. Moreover, as stated in 
TABLE 7: Numerical natural frequencies (in $\%$ of $f_{\max }$ ) estimated by the FE model regarding the modified drivelines. Italic characters highlight frequencies that fall in the frequency band of interest.

(a) Simulation results 45 Shore MOD 1

\begin{tabular}{lc}
\hline Mode 1 & $2.5 \%[1 \mathrm{st} \mathrm{TG}]$ \\
Mode 2 & $6.1 \%[1 \mathrm{st} \mathrm{LC}]$ \\
Mode 3 & $8 \%[2 \mathrm{nd} \mathrm{TG}]$ \\
Mode 4 & $13.8 \%[2 \mathrm{nd} \mathrm{LC}]$ \\
Mode 5 & $17.8 \%[3 \mathrm{rd} \mathrm{LC}]$ \\
Mode 6 & $26.1 \%[1$ st AF] \\
Mode 7 & $72.2 \%[3 \mathrm{rd} \mathrm{TG}]$ \\
Mode 8 & $98.6 \%[1 \mathrm{st} \mathrm{BS}]$ \\
\hline
\end{tabular}

(b) Simulation results 70 Shore MOD 2

\begin{tabular}{cc}
\hline Mode 1 & $4.5 \%[1$ st TG] \\
Mode 2 & $13.4 \%[1 \mathrm{st} \mathrm{LC}]$ \\
Mode 3 & $24.3 \%[2 n d$ TG] \\
Mode 4 & $33.7 \%[2 n d ~ L C]$ \\
Mode 5 & $60.9 \%[3 \mathrm{rd} \mathrm{LC}]$ \\
Mode 6 & $73.1 \%[1 \mathrm{st} \mathrm{AF}]$ \\
Mode 7 & $80.4 \%[3 \mathrm{rd} \mathrm{TG}]$ \\
\hline
\end{tabular}

(c) Simulation results 60 Shore MOD 3

\begin{tabular}{lc}
\hline Mode 1 & $3.7 \%[1$ st TG] \\
Mode 2 & $11.5 \%[1 \mathrm{st} \mathrm{LC}]$ \\
Mode 3 & $24.9 \%[2 n d$ TG] \\
Mode 4 & $32.7 \%[2 n d$ LC] \\
Mode 5 & $62.2 \%[3 \mathrm{rd} \mathrm{LC}]$ \\
Mode 6 & $73.1 \%[1 \mathrm{st} \mathrm{AF}]$ \\
Mode 7 & $77.6 \%[3 \mathrm{rd} \mathrm{TG}]$ \\
\hline
\end{tabular}

Section 3.2, the second local mode of the coupling (2nd LC) is not excited in operational conditions. The quality of these improvements can be appreciated also in Section 3.6.

3.5. Elastodynamic Analysis: Model Validation. It has to be highlighted that since in the frequency range of interest only pure-torsional modes occur (as well as not-excited axial and local modes, see Section 3.3), the elastodynamic model was focused on the torsional dynamics only.

The validation of the elastodynamic model described in Section 2.5 was based on the experimental analysis results of Sections 3.1 and 3.2. It should be noted that experimental data collected in the $45 \mathrm{Sh}$ rubber element configuration could not be used for the validation of such a model. In fact, those data are mainly affected by the presence of the local mode of the coupling, which cannot be predicted by the pure-torsional model described above. The model validation was thus performed on data acquired with the 70 Sh rubber coupling.

The natural frequencies computed by the LP model are in good accordance with those from FE model and experimental activity. The comparison is shown in Table 8. The output parameter for both experimental data and simulation results is the Irregularity Ratio (IR), between the $n_{\text {rel }}$ peak-to-peak
TABLE 8: Comparison of resonance frequencies in (a) experimental measurements, (b) FE model, and (c) LP model for the 70 Sh rubber element configuration with reference to $f_{\max }$.

(a) Experimental resonances

\begin{tabular}{lc}
\hline Mode 1 & $6 \%-8 \%$ \\
Mode 2 & $24 \%-30 \%$ \\
\hline
\end{tabular}

(b) FE results

\begin{tabular}{cc}
\hline Mode 1 & $5.1 \%[1$ st TG] \\
Mode 2 & $13 \%[1$ st LC] \\
Mode 3 & $25.7 \%[2 \mathrm{nd} \mathrm{TG}]$ \\
Mode 4 & $37.2 \%[2 \mathrm{nd} \mathrm{LC}]$ \\
Mode 5 & $60.2 \%[3 \mathrm{rd} \mathrm{TG}]$ \\
Mode 6 & $60.4 \%[3 \mathrm{rd} \mathrm{LC}]$ \\
Mode 7 & $83.6 \%[1 \mathrm{st} \mathrm{AF}]$ \\
Mode 8 & $85.1 \%[1 \mathrm{st} \mathrm{BS}]$ \\
\hline
\end{tabular}

(c) LP results

\begin{tabular}{lc}
\hline Mode 1 & $4.5 \%$ \\
Mode 2 & $24.9 \%$ \\
Mode 3 & $58.2 \%$ \\
\hline
\end{tabular}

amplitude and the mean value of the transmission shaft velocity, expressed as percentage. Results in correspondence of the rotational coordinates at different speed of the engine are shown in Table 9.

Simulation results are in good accordance with the experimental data, both clearly identifying a higher oscillation at Regime A that decreases as the speed increases from A to E, and then it increases again at Regime F. The advantage of the elastodynamic model is that the causes of the increased oscillation could be easily investigated through the simulation. In particular, the analysis of the resonance frequencies of the system shows that mode shape 2 is particularly burdensome for coordinate $\theta_{\mathrm{mf}}$ (Figure 12). The corresponding natural frequency is quite close to the 1st crankshaft order of Regime A, thus justifying both simulation results and experimental analysis. Moreover, the same natural frequency is still close to the half of the crankshaft order of Regime F.

3.6. Elastodynamic Analysis: Simulation Results and Discussion. The elastodynamic model of the driveline was used to simulate the dynamic behavior of the system in three different design changes. The same designation of the modifications discussed in Section 3.4 is used. Results are given as Irregularity Ratio: Tables 10, 11, and 12 collect the IR for MOD 1, MOD 2, and MOD 3.

The power loss was used as a further indicator. According to standard DIN 740 the relative damping is the ratio between the power loss of one vibration cycle and elastic deformation energy. The elastic deformation energy depends on the main frequency in the oscillation spectrum of the coupling and can be easily computed, while the relative damping is usually given in the manufacturer's catalog (it is the $\gamma$ parameter in (2)). The power losses of one vibration cycle for different 
TABLE 9: Comparison between simulation results and experimental data, as IR values.

\begin{tabular}{lcccccc}
\hline Simulation & Regime A & Regime B & Regime C & Regime D & Regime E & Regime F \\
\hline $\mathrm{IR}_{c}$ & 4.5 & 3.5 & 2.9 & 2.5 & 2.1 & 3.3 \\
$\mathrm{IR}_{\mathrm{es}}$ & 4.9 & 2 & 2.2 & 2.7 & 1.7 & 3.4 \\
$\mathrm{IR}$ & 10.3 & 3.3 & 2.0 & Regime D & Regime E & Regime F \\
\hline Experiment & Regime A & Regime B & Regime C & 2.9 & 2.5 & 2.1 \\
\hline $\mathrm{IR}_{c}$ & 5.4 & 4.3 & 3.5 & 2.4 & 1.8 & 3.2 \\
$\mathrm{IR}_{\mathrm{es}}$ & 7.9 & 4.0 & 2.9 & & & \\
\hline
\end{tabular}

TABLE 10: Simulation results of MOD 1.

\begin{tabular}{lcccccc}
\hline MOD 1 & Regime A & Regime B & Regime C & Regime D & Regime E & Regime F \\
\hline $\mathrm{IR}_{c}$ & 2.4 & 2.1 & 1.8 & 1.3 & 0.9 & 0.7 \\
$\mathrm{IR}_{\mathrm{es}}$ & 3.4 & 4.2 & 1.9 & 0.13 .2 & 1.4 \\
$\mathrm{IR}_{\mathrm{mf}}$ & 0.30 & 0.19 & 0.07 & 0.04 & 0.03 \\
\hline
\end{tabular}

TABLE 11: Simulation results of MOD 2.

\begin{tabular}{lcccccc}
\hline MOD 2 & Regime A & Regime B & Regime C & Regime D & Regime E & Regime F \\
\hline $\mathrm{IR}_{c}$ & 2.4 & 2.1 & 1.8 & 1.3 & 1.0 & 0.7 \\
$\mathrm{IR}_{\mathrm{es}}$ & 2.8 & 2.7 & 2.2 & 1.4 & 1.8 & 1.1 \\
$\mathrm{IR}_{\mathrm{mf}}$ & 3.24 & 1.55 & 1.14 & 1.04 & 2.09 & 0.35 \\
\hline
\end{tabular}

TABLE 12: Simulation results of MOD 3.

\begin{tabular}{lcccccc}
\hline MOD 3 & Regime A & Regime B & Regime C & Regime D & Regime E & Regime F \\
\hline $\mathrm{IR}_{c}$ & 2.4 & 2.0 & 1.7 & 1.3 & 1.0 & 0.7 \\
$\mathrm{IR}_{\mathrm{es}}$ & 2.7 & 4.4 & 2.0 & 1.5 & 1.19 & 1.0 \\
$\mathrm{IR}$ & 2.82 & 1.43 & 1.16 & 1.51 & 0.32 \\
\hline
\end{tabular}
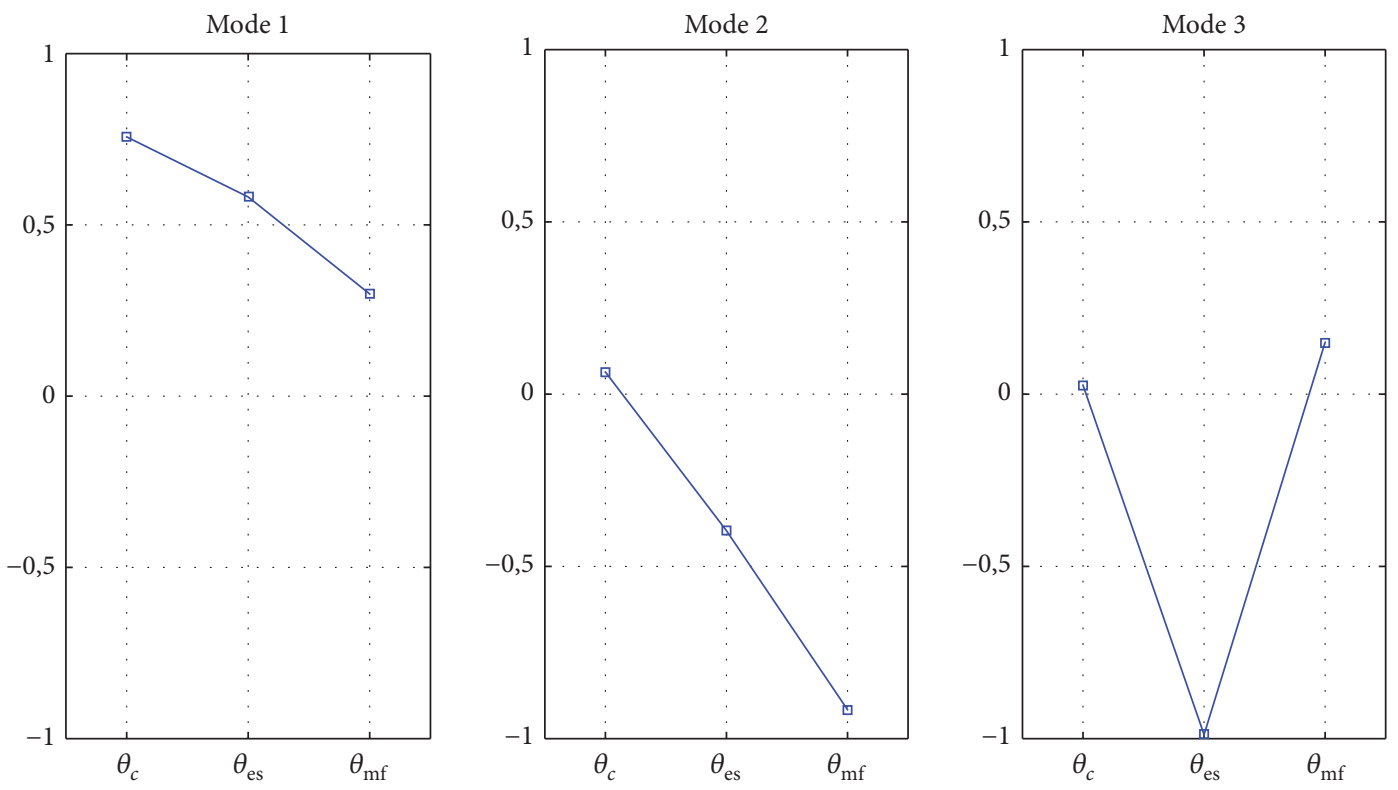

FIGURE 12: Normalized mode shapes of the driveline. 
TABLE 13: The power loss of one vibration cycle for each design modification.

\begin{tabular}{lcccccc}
\hline$[\mathrm{W}]$ & Regime A & Regime B & Regime C & Regime D & Regime E & Regime F \\
\hline MOD 1 & 1.03 & 1.23 & 0.72 & 0.99 & 4.98 & 0.87 \\
MOD 2 & 31.87 & 13.18 & 4.80 & 3.54 & 2.90 & 2.66 \\
MOD 3 & 19.14 & 9.76 & 3.83 & .88 & 1.89 \\
\hline
\end{tabular}

hardness of the rubber and different speeds are collected in Table 13.

Modification MOD 1 keeps torsional vibrations at low level, with a stable and limited oscillation around the reference speed. Consequently, the power loss has low values compared to the other modifications. As a drawback, the FE analysis shows that all the resonance frequencies are shifted to lower values; for example, five resonances lay in the band $\left(0 \%-18 \%\right.$ of $\left.f_{\max }\right)$. Even if they are outside the frequency band of interest $\left(18 \%-60 \%\right.$ of $\left.f_{\max }\right)$, it is not excluded that the test rig will be used at lower speed regimes in the future, with the consequent need of further design modifications to avoid resonance problems again. Moreover, the MOD 1 requires a sensible increase of the coupling's mass and then a further structural load on the supports of the test rig.

Modifications MOD 2 and MOD 3 are in the opposite direction of MOD 1 . They shifted the resonance frequencies at higher values out of the selected frequency bandwidth. Torsional vibrations are still acceptable: the speed oscillation is less than 5\% with respect to regime. In MOD 2 the FE model shows that the 3rd local mode frequency is outside the limit of $60 \%$ of $f_{\max }$ but still close (60.9\%), while MOD 3 takes a little bit higher safety factor (62.2\%). Comparing the damping power in Table 13, MOD 2 shows an increased value at Regimes A and B-probably due to a close resonance-which is reduced in MOD 3. These considerations led to choose MOD 3 as the optimal design improvement.

\section{Conclusions}

The paper presents an effective methodology based on numerical models and experimental measurements, which aims at foreseeing the dynamic behavior of highly flexible couplings of IC engine test rigs in real operational conditions. The main goal is to prevent high level of torsional oscillations, whirling of coupling shafts, and early failures of any components. A procedure to optimize the dynamic behavior of the driveline is thus proposed and applied to an industrial application, useful to show that fully understanding of the dynamic behavior of a real mechanical system requires more than one modeling approach besides an experimental activity. In the test bench being studied, the output shaft of the engine is connected to an electromechanical brake through a transmission shaft, which hosts a highly flexible coupling with rubber elements.

The results of the reported case study led to draw the following conclusions:

(i) the experimental activity showed the presence of a resonance close to the working condition of the coupling, which led to an early breaking of the rubber elements of the joint; (ii) the FE model enabled the characterization of the shape of the mode determining such a resonance and the suggestion of three different design modifications to avoid the resonance in working conditions;

(iii) the LP model allowed choosing the design modification less burdensome in terms of torsional vibration of the driveline.

The presented methodology could be a very useful tool in prototype design and optimization, as well as identifying the origin of unwanted dynamic effects in test rig. Although these quantitative results concern a particular test rig, the used methodology and the drawn conclusions have a general meaning from the qualitative point of view. They can be thus applied to a large variety of mechanical systems and applications offering useful guidelines in order to foresee the influence of operational conditions and design modifications on vibration generation.

\section{Nomenclature}

$P_{i}: \quad$ Pressure in $i$-th cylinder

$d: \quad$ Bore of the piston

$r$ : $\quad$ Length of the crank

$m_{p}:$ Mass of the piston

COG: Center of gravity

$m_{b}$ : Mass of the piston rod (p.r.)

$l: \quad$ Length of the p.r.

$J_{b}: \quad$ Inertia of the p.r.

$l_{a}$ : $\quad$ Distance COG and head of p.r.

$l_{b}$ : $\quad$ Distance COG and foot of p.r.

\section{Conflicts of Interest}

The authors declare that they have no conflicts of interest.

\section{Acknowledgments}

This work has been developed within the Advanced Mechanics Laboratory (MechLav) of Ferrara Technopole, realized through the contribution of Regione Emilia-RomagnaAssessorato Attività Produttive, Sviluppo Economico, Piano telematico-POR-FESR 2007-2013, Activity I.1.1.

\section{References}

[1] E. M. A. Rabeih and D. A. Crolla, "Coupling of driveline and body vibrations in trucks," in Proceedings of the 1996 SAE International Truck and Bus Meeting and Exposition, vol. 1203, SAE Conf. Trans., Detroit, 1996.

[2] A. Reitz, J. W. Biermann, and P. Kelly, "Special test benches to investigates driveline related NVH phenomena," 8th Aachen Colloquium, Automobile and engine Technology, 1999. 
[3] S. Gequn, L. Min, and W. Haiqiao, "Research on the influence of bench installation conditions on simulation of engine main bearing load," SAE InternatIonal Journal of Engines, pp. 18851890, 2009.

[4] D. L. Dewell and L. D. Mitchell, "Detection of a misaligned disk coupling using spectrum analysis," Journal of Vibration, Acoustics, Stress, and Reliability in Design, vol. 106, no. 1, pp. 9$16,1984$.

[5] H.-W. Cho and M. K. Jeong, "Enhanced prediction of misalignment conditions from spectral data using feature selection and filtering," Expert Systems with Applications, vol. 35, no. 1-2, pp. 451-458, 2008.

[6] T. H. Patel and A. K. Darpe, "Experimental investigations on vibration response of misaligned rotors," Mechanical Systems and Signal Processing, vol. 23, no. 7, pp. 2236-2252, 2009.

[7] M. Xu and R. D. Marangoni, "Vibration analysis of a motorflexible coupling-rotor system subject to misalignment and unbalance, part I: theoretical model and analysis," Journal of Sound and Vibration, vol. 176, no. 5, pp. 663-679, 1994.

[8] F. Cruz-Peragón, J. M. Palomar, F. A. Díaz, and F. J. JiménezEspadafor, "Practical identification of non-linear characteristics of elastomeric couplings in engine assemblies," Mechanical Systems and Signal Processing, vol. 23, no. 3, pp. 922-930, 2009.

[9] M. Troncossi, E. Mucchi, and A. Rivola, “Torsional Vibration Analysis of a Test Rig Driveline Equipped with a Flexible Coupling," in Proceedings of 5th European Conference of Mechanical Engineering (ECME '14, pp. 198-206, Florence (Italy, 2014.

[10] K. Janssens, P. Van Vlierberghe, W. Claes, B. Peeters, T. Martens, and P. D'Hondt, "Zebra tape butt joint detection and correction algorithm for rotating shafts with torsional vibrations," in Proceedings of the ISMA2010, Leuven, Belgium, September 2022, 2010.

[11] K. R. Fyfe and E. D. S. Munck, "Analysis of computed order tracking," Mechanical Systems and Signal Processing, vol. 11, no. 2, pp. 187-202, 1997.

[12] S. Gade, H. Herlufsen, H. Konstantin-Hansen, and N. J. Wismer, "Order Tracking Analysis," Technical Review No. 2, Brüel \& Kjær, 1995.

[13] S. Braun, "The synchronous (time domain) average revisited," Mechanical Systems and Signal Processing, vol. 25, no. 4, pp. 1087-1102, 2011.

[14] A. V. Oppenheim, R. W. Schafer, and J. R. Buck, Discrete-Time Signal Processing, Prentice-Hall, 1999.

[15] R. X. Gao and R. Yan, "From fourier transform to wavelet transform: a historical perspective," in Wavelets - Theory and Applications for Manufacturing (Chapter 2), Springer, BerlinHeidelberg, 2011.

[16] E. Mucchi, G. Bottoni, and R. Di Gregorio, "Indirect measurement of the inertia properties of a knee prosthesis through a simple frequency-domain technique," Journal of Medical Devices, vol. 3, Article ID 044501-5, 2009.

[17] E. Mucchi, S. Fiorati, R. Di Gregorio, and G. Dalpiaz, "Determining the rigid-body inertia properties of cumbersome systems: Comparison of techniques in time and frequency domain," Experimental Techniques, vol. 35, no. 3, pp. 36-43, 2011.

[18] E. Ciulli, "A review of internal combustion engine losses. part 2: studies for global evaluations," ARCHIVE: Proceedings of the Institution of Mechanical Engineers, Part D: Journal of Automobile Engineering 1989-1996 (vols 203-210), vol. 207, no. 3, pp. 229-240, 1993.
[19] D. E. Richardson, "Review of power cylinder friction for diesel engines," Journal of Engineering for Gas Turbines and Power, vol. 122, pp. 608-618, 2000.

[20] J. B. Heywood, Internal Combustion Engine Fundamentals, vol. 26, McGraw-Hill, New York, USA, 1988.

[21] K. Liu, X. J. Liu, and C. L. Gui, "Scuffing failure analysis and experimental simulation of piston ring-cylinder liner," Tribology Letters, vol. 5, no. 4, pp. 309-312, 1998.

[22] H. M. Uras and D. J. Patterson, "Measurement of piston and ring assembly friction instantaneous IMEP method," SAE Paper, Article ID 830416, 1983.

[23] S. F. Rezeka and N. A. Henein, "A new approach to evaluate instantaneous friction and its components in internal combustion engines," SAE Technical Papers, Article ID 840179, 1985.

[24] N. G. Chalhoub, H. Nehme, N. A. Henein, and W. Bryzik, "Effects of structural deformations of the crank-slider mechanism on the estimation of the instantaneous engine friction torque," Journal of Sound and Vibration, vol. 224, no. 3, pp. 489503, 1999.

[25] Y. H. Zweiri, J. F. Whidborne, and L. D. Seneviratne, "Instantaneous friction components model for transient engine operation," Proceedings of the Institution of Mechanical Engineers, Part D: Journal of Automobile Engineering, vol. 214, no. 7, pp. 809$824,2000$.

[26] Y. H. Zweiri, J. F. Whidborne, and L. D. Seneviratne, "Detailed analytical model of a single-cylinder diesel engine in the crank angle domain," Proceedings of the Institution of Mechanical Engineers, Part D: Journal of Automobile Engineering, vol. 215, no. 11, pp. 1197-1216, 2001.

[27] F. Cruz-Peragón, J. M. Palomar, F. A. Díaz, and F. J. Jiménez-Espadafor, "Fast on-line identification of instantaneous mechanical losses in internal combustion engines," Mechanical Systems and Signal Processing, vol. 24, no. 1, pp. 267-280, 2010.

[28] H. J. Qi and M. C. Boyce, "Stress-strain behavior of thermoplastic polyurethanes," Mechanics of Materials, vol. 37, no. 8, pp. 817839, 2005.

[29] C. Y. Wei and S. N. Kukureka, "Evaluation of damping and elastic properties of composites and composite structures by the resonance technique," Journal of Materials Science, vol. 35, no. 15, pp. 3785-3792, 2000.

[30] S. Y. Chen, M. S. Ju, and Y. G. Tsuei, "Estimation of mass, stiffness and damping matrices from frequency response functions," Journal of Vibration and Acoustics, vol. 118, no. 1, pp. 78-82, 1996.

[31] S. S. Rao, Mechanical Vibrations, Prentice Hall, New York, 2003.

[32] J. Uicker, G. Pennock, and J. Shigley, Theory of Machines and Mechanisms, Oxford University Press, New York, USA, 2010.

[33] M. Cocconcelli, A. Agazzi, E. Mucchi, G. Dalpiaz, and R. Rubini, "Dynamic analysis of coupling elements in IC engine test rigs," in Proceedings of the ISMA2014 - USD2014 Conference, pp. 10051018, Leuven, Belgium, September 2014. 


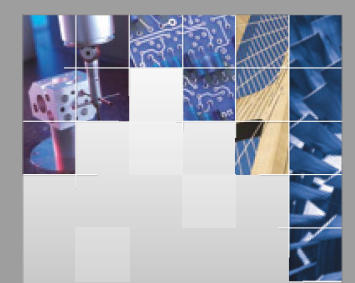

\section{Enfincering}
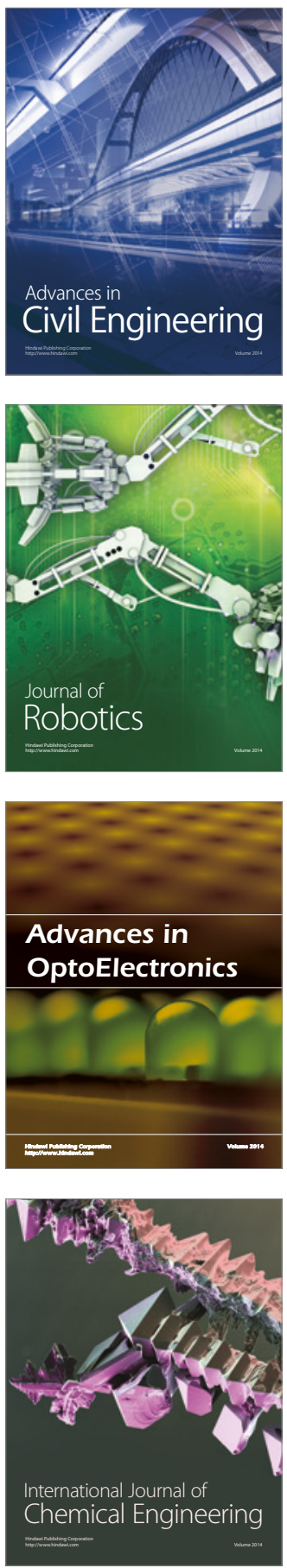

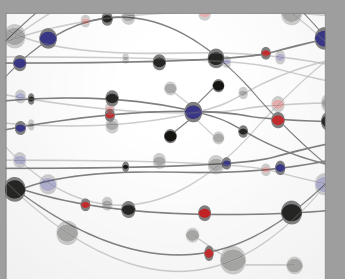

The Scientific World Journal

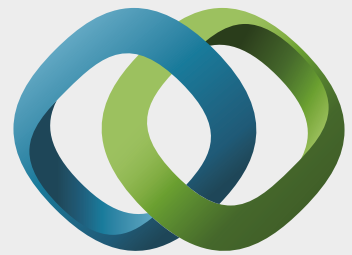

\section{Hindawi}

Submit your manuscripts at

https://www.hindawi.com
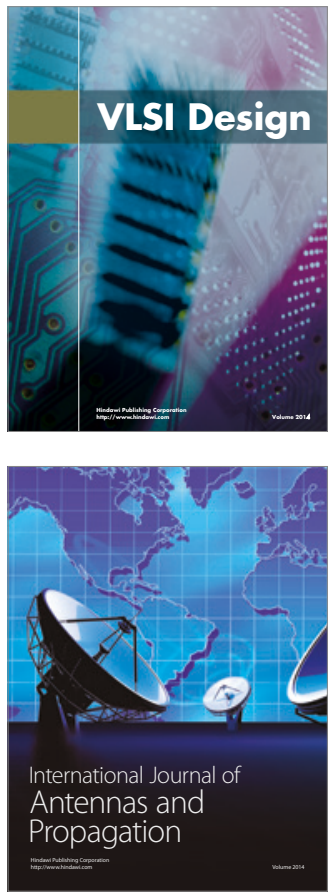

\section{Rotating}

Machinery
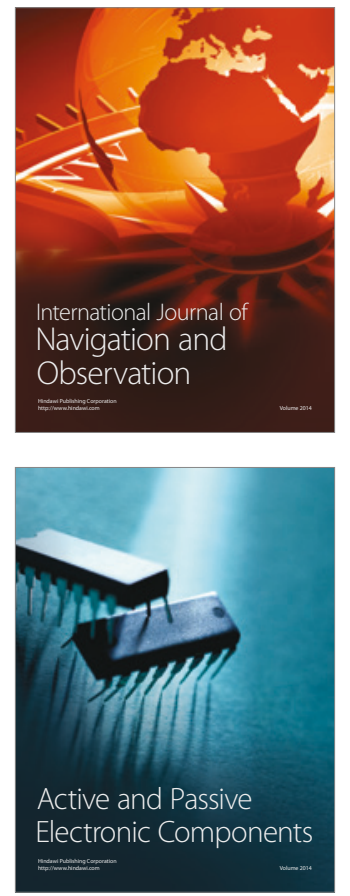
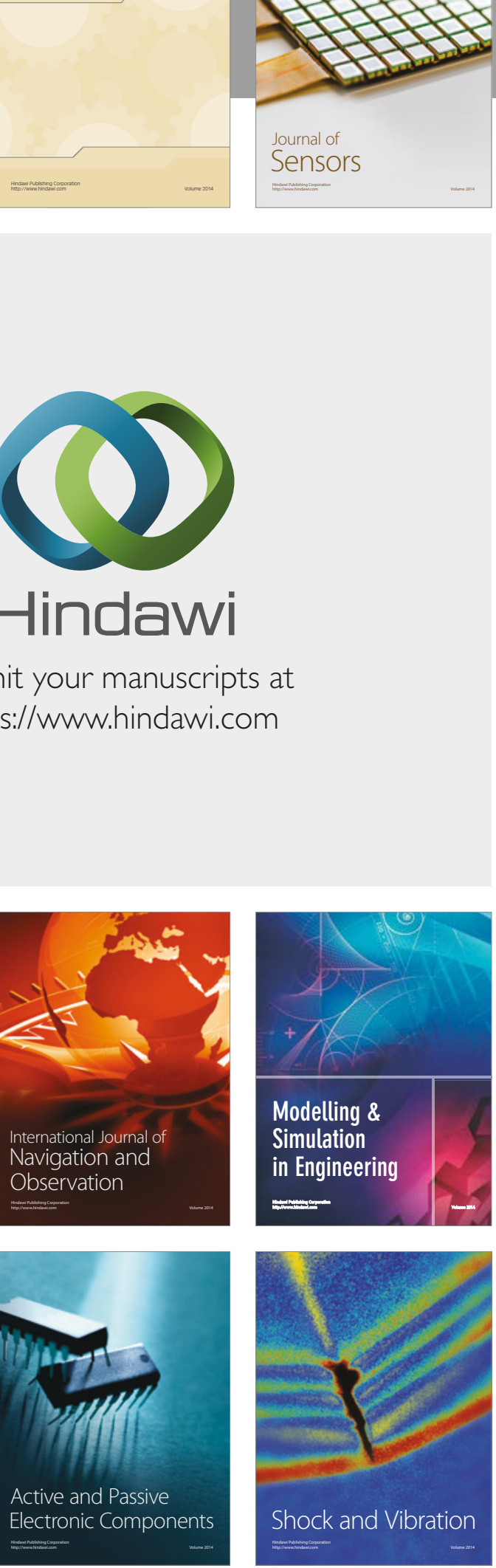
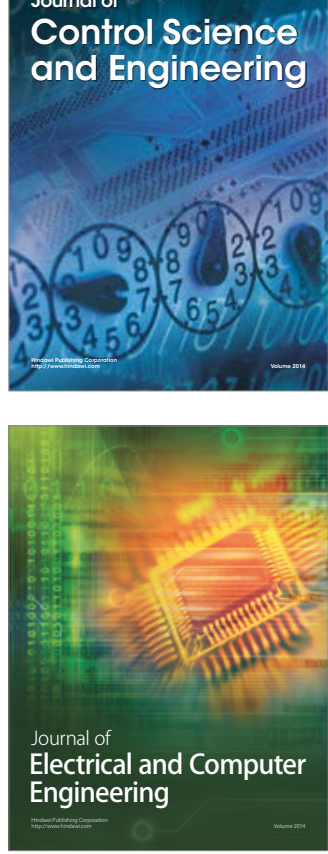

Distributed

Journal of

Control Science

and Engineering
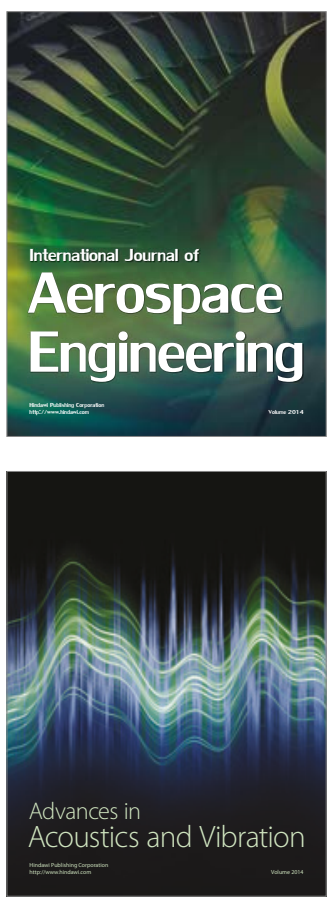

Sensor Networks 